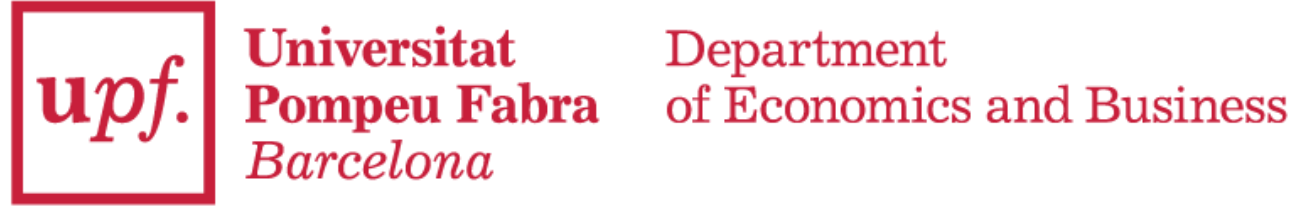

Economics Working Paper Series

Working Paper No. 1555

\title{
Understanding the size of the government spending multiplier: It's in the sign
}

\author{
Regis Barnichon \\ Christian Matthes
}

May 2016 


\title{
Understanding the Size of the Government Spending Multiplier: It's in the Sign*
}

\author{
Regis Barnichon \\ CREI, Universitat Pompeu Fabra, CEPR \\ Christian Matthes \\ Federal Reserve Bank of Richmond
}

May 2016

\begin{abstract}
Despite intense scrutiny, estimates of the government spending multiplier remain highly uncertain, with values ranging from 0.5 to 2 . While an increase in government spending is generally assumed to have the same (mirror-image) effect as a decrease in government spending, we show that relaxing this assumption is important to understand the effects of fiscal policy. Regardless of whether we identify government spending shocks from (i) a narrative approach, or (ii) a timing restriction, we find that the contractionary multiplier -the multiplier associated with a negative shock to government spending- is above 1, while the expansionary multiplier - the multiplier associated with a positive shock- is substantially below 1 . The multiplier is largest in recessions, as found in previous studies, but only because the contractionary multiplier is largest in recessions. The expansionary multiplier is always below 1 and not larger in recessions. We argue that our results help understand the wide range of multiplier estimates found in the literature.
\end{abstract}

(May 2016

JEL classifications: C32, E62

\footnotetext{
${ }^{*}$ We would like to thank Davide Debortoli, Emmanuel Fahri, Jordi Gali, Yuriy Gorodnichenko, Oscar Jorda, Peter Karadi, Karel Mertens, Silvana Tenreyro, Helene Rey and seminar participants for helpful comments. We thank Alexander Ziegenbein for superb research assistance. Barnichon acknowledges financial support from the Spanish Ministerio de Economia y Competitividad (grant ECO2011-23188) and the Barcelona GSE Research Network. The views expressed here do not necessarily reflect those of the Federal Reserve Bank of Richmond or the Federal Reserve System. Any errors are our own.
} 


\section{Introduction}

Understanding the impact of expansionary fiscal policy on output is a central part of fiscal policy analysis, and the question received much attention as many OECD countries implemented fiscal stimulus packages during the early stages of the 2008-2009 recession. ${ }^{1}$ As government debt levels rose rapidly, a swift shift to fiscal consolidation followed (particularly in continental Europe) and spurred work on the mirror-image question - the effect of contractionary fiscal policy-. ${ }^{2}$ However despite intense scrutiny, the range of estimates for the government spending multiplier remains wide with estimates lying between 0.5 and 2 .

Perhaps surprisingly, the literature has so far treated the two aforementioned questions symmetrically: a contractionary policy is assumed to have the same (mirror-image) effect as an expansionary policy, and previous studies on the government spending multiplier have treated symmetrically the effects of contractionary and expansionary government spending shocks.

In this paper, we relax this assumption using a novel econometric procedure, and we find that treating separately expansionary and contractionary spending shocks is important to (i) estimate the size of the government spending multiplier, and (ii) understand the wide range of estimates across studies.

We obtain three main results. First, the effect of government spending on economic activity is highly asymmetric, and the size of the government spending multiplier depends crucially on the sign of the fiscal intervention: the government spending multiplier is substantially below 1 for expansionary shocks to government spending, but the multiplier is above 1 for contractionary shocks. Second, the asymmetric effect of government spending is related to the strong asymmetric response of investment: While a contractionary spending shock generates a significant drop in investment, an expansionary government spending shock crowds

\footnotetext{
${ }^{1}$ See e.g., Hall (2009), Ramey (2009), Mertens and Ravn (2010), Barro and Redlick (2011), Parker (2011), Ramey (2011, 2012a, 2012b), Auerbach and Gorodnichenko (2012, 2013), Owyang, Ramey and Zubairy (2013) and Ramey and Zubairy (2014), Caggiano, Castelnuovo, Colombo and Nodari (2015).

${ }^{2}$ See e.g., Alesina and Ardagna (2010), Guajardo, Leigh, and Pescatori (2011) and Jorda and Taylor (2013).
} 
out investment and thus also generates a significant drop in investment. In other words, any shock to government spending appears to negatively affect private investment. ${ }^{3}$ Third, the multiplier associated with contractionary shocks to government spending is state-dependent -being largest and around 2 in recessions-, but the multiplier associated with expansionary shocks to government spending is not state-dependent, -being always below 1 and not larger in recessions-.

Importantly, we reach these same three conclusions regardless of whether we identify government spending shocks from -(i) a narrative identifying assumption (Ramey, 2011), or (ii) a recursive identifying assumption (Blanchard and Perotti, 2002, Auerbach and Gorodnichenko, 2012)-, which have been the two main approaches to identifying government spending shocks and their effects.

Treating expansionary and contractionary shocks separately also helps to reconcile seemingly contradictory findings in the literature on government multipliers: While estimates from shocks to defense spending (Barro and Redlick 2011, Ramey and Zubairy 2014) are significantly less than 1, estimates from shocks recovered from Vector AutoRegressions (VARs) are often close to or above 1 (although with some dispersion across studies), ${ }^{4}$ and studies of fiscal consolidations (Jorda and Taylor, 2013) can find even larger multipliers with values significantly above 1 and closer to 2 .

We argue that these different results stem in part from variations in the sample of fiscal shocks recovered by each method and more specifically from variations in the relative frequency of expansionary and contractionary shocks. Results obtained from shocks to defense spending are driven primarily by positive shocks - unexpected increases in government spending-, because the narratively-identified shock series (Ramey, 2011) contains much more positive shocks than negative shocks. As a result, the estimated multiplier is small, because the multiplier associated with positive shocks is small. In contrast, the spending shocks identified in VARs

\footnotetext{
${ }^{3} \mathrm{An}$ interesting corollary is that an increase in the variance of government spending shocks, i.e., an increase in the uncertainty associated with the path of government spending lowers private investment.

${ }^{4}$ See for instance Blanchard and Perotti (2002), Fisher and Peters (2010), Auerbach and Gorodnichenko (2012), Gordon and Krenn (2010), and Ben Zeev and Pappa (2015).
} 
are (by construction) evenly distributed between positive and negative values. As a result, the multiplier is larger, lying in between our estimates of the expansionary multiplier and the contractionary multiplier. Finally, Jorda and Taylor (2013)'s estimates are much larger than the other lines of work simply because they strictly focus on fiscal consolidations. Their estimated multiplier is thus that of a contractionary multiplier, which we also find to be large.

A similar reasoning can help reconcile seemingly contradictory estimates for state dependence. While studies based on narratively-identified shocks (Owyang, Ramey and Zubairy 2013, Ramey and Zubairy 2014) find little evidence for state dependence, VAR-based studies such as Auerbach and Gorodnichenko (2012) find strong evidence for state dependence. Again, the different distributions of shocks across studies can rationalize these conflicting results. Since narrative-studies are dominated by positive shocks to government spending, the results should mostly reflect the effects of positive shocks, which (according to our results) do not depend on the state of the business cycle. In contrast, since VAR-based studies have similar distributions of positive and negative shocks, the results should reflect the fact that (according to our results) negative shocks have state-dependent effects. Interestingly, while Auerbach and Gorodnichenko (2012)'s findings have sometimes been interpreted as supporting the case for fiscal stimulus in recessions, our results caution against such a conclusion. We find no evidence that increases in government spending have larger multipliers during a recession (in fact, the multiplier is consistently below 1) and thus no support for stimulus programs in times of recession. However, we find that contractionary government spending shocks during recessions have the largest multiplier, which suggests that austerity measures during recessions can be especially harmful.

Part of the reason for the lack of studies on the possibly asymmetric (and state dependent) nature of the government spending multiplier is methodological. On the one hand, standard techniques are linear and make the exploration of non-linearities, in particular the asymmetric effect of spending shocks and their state dependence, difficult. Structural VARs, as used by Blanchard and Perotti (2002) and Auerbach and Gorodnichenko (2012) are ill-suited to allow 
the impulse response function of a shock to depend on the sign of that shock, because in such cases the existence of a VAR representation is compromised. ${ }^{5}$ On the other hand, the narrative approach to government spending shocks, pioneered by Ramey and Shapiro (1998) and Ramey (2011), relies on autoregressive distributed lags (ADL) models or Local Projection (LP, Jorda, 2005), and these methods can allow for some non-linearities, as illustrated by Ramey and Zubairy (2013) and Auerbach and Gorodnichenko (2013) for state dependence. However, because of their non-parametric nature, these methods are limited by efficiency considerations, and simultaneously allowing for asymmetry and state dependence is difficult. ${ }^{6}$

To overcome these technical challenges, we use a new method that consists in (i) directly estimating a structural moving average model of the economy, i.e., directly estimating the impulse response functions to structural shocks (unlike the VAR approach, which first estimates a reduced-form VAR and thus requires the existence of a VAR representation), and (ii) parameterizing the impulse response functions with a small number of Gaussian functions, which offers efficiency gains and allows for the exploration of a rich set of non-linearities (unlike the non parametric ADL or LP approach).

Our Gaussian Mixture Approximation (GMA) of impulse responses builds on two premises: (i) any mean-reverting impulse response function can be approximated by a mixture of Gaussian basis functions, and (ii) a small number (one or two) of Gaussian functions can already cap-

\footnotetext{
${ }^{5}$ Regime-switching VAR models can capture certain types of non-linearities such as state dependence (whereby the value of some state variable affects the impulse response functions), but they cannot capture asymmetric effects of shocks (whereby the impulse response to a structural shock depends on the sign of that shock). With regime-switching VAR models, it is assumed that the economy can be in a finite number of regimes, and that each regime corresponds to a different set of VAR coefficients. However, if the true data generating process features asymmetric impulse responses, a new set of VAR coefficients would be necessary each period, because the (non-linear) behavior of the economy at any point in time depends on all structural shocks up to that point. As a result, such asymmetric data generating process cannot generally be approximated by a small number of state variables such as in threshold VARs or Markov-switching models. In contrast, by working directly with the structural moving-average representation, GMA models can easily capture asymmetric impulse response functions (as well as state dependence).

${ }^{6}$ Riera-Crichton et al. (2015) use Jorda's local projection method to study the state dependent response of output growth to positive and negative government spending shocks. However because of efficiency considerations, they can only consider two states (expansion vs recession) and they must rely on cross-country data to bring in additional information. Specifically, they use a semiannual sample of 30 OECD countries that starts in 1985 at the earliest and impose that responses are the same across countries. Thanks to our more efficient procedure, we can focus on a single country, so that our results are not contaminated by cross-country heterogeneity.
} 
ture a large variety of impulse response functions, and in fact capture the typical impulse responses found in empirical or theoretical studies. For instance, the impulse response functions of macroeconomic variables to government spending shocks are often found (or predicted) to be monotonic or hump-shaped (e.g. Ramey, 2011, Gali et al., 2007). In such cases, a single Gaussian function can already provide an excellent approximation of the impulse response function. Thanks to the small number of free parameters allowed by our Gaussian mixture approximation, it is possible to directly estimate the impulse response functions from the data using maximum likelihood or Bayesian methods. The parsimony of the approach in turn allows us to estimate more general non-linear models.

Our use of Gaussian functions to approximate (and parametrize) impulse response functions builds on Barnichon and Matthes (2016) and relates to a large literature outside of economics that relies on radial basis functions (of which Gaussian functions are one example) to approximate arbitrary multivariate functions (e.g., Buhmann, 2003) or to approximate arbitrary distributions using a mixture of Gaussian distributions (Alspach and Sorenson 1971, 1972, McLachlan and Peel, 2000). In economics, our parametrization of impulse responses relates to an older literature on distributed lag models and in particular on the Almon (1965) lag specification, in which the successive weights, i.e., the impulse response function in our context, are given by a polynomial function.

Section 2 presents the empirical model, our method to approximate impulse responses using Gaussian basis functions and the two main structural identifying restrictions used in the literature, Section 3 describes our asymmetric GMA model and presents our results on the asymmetric effects of shocks to government spending; Section 4 describes a GMA model with asymmetry and state dependence, presents the results and discusses how the asymmetric effects of government spending shocks helps reconcile the seemingly contradictory findings previously reported in the literature; Section 5 concludes. 


\section{Empirical model}

Our goal in this paper is to study to what extent the size of the government spending multiplier, and more generally the effects of government spending on the economy, depends on the sign of the policy intervention and on the state of the business cycle at the time of the policy intervention.

To capture these possibilities, we need a model that allows the impulse response functions to depend on the sign of the shock as well as on the state of the economy at the time of the shock. ${ }^{7}$ Our empirical model is thus a (non-linear) structural moving-average model, in which the behavior of a vector of macroeconomic variables is dictated by its response to past and present structural shocks. Specifically, denoting $\boldsymbol{y}_{t}$ a vector of stationary macroeconomic variables, the economy is described by

$$
\boldsymbol{y}_{t}=\sum_{k=0}^{K} \boldsymbol{\Psi}_{k}\left(\varepsilon_{t-k}, z_{t-k}\right) \varepsilon_{t-k}
$$

where $\varepsilon_{t}$ is the vector of structural innovations with $E \varepsilon_{t}=\mathbf{0}$ and $E \varepsilon_{t} \varepsilon_{t}^{\prime}=\mathbf{I}, K$ is the number of lags, which can be finite or infinite, $z_{t}$ is a stationary variable that is a function of lagged values of $\boldsymbol{y}_{t}$ or a function of variables exogenous to $\boldsymbol{y}_{t} \cdot \boldsymbol{\Psi}_{k}$ is the matrix of lag coefficients -i.e., the matrix of impulse responses at horizon $k^{-}$.

Model (1) is a non-linear vector moving average representation of the economy, because the matrix of lag coefficients $\boldsymbol{\Psi}_{k}$, i.e., the impulse responses of the economy, can depend on (i) the values of the structural innovations $\varepsilon$ and (ii) the value of the macroeconomic variable $z$ : With $\Psi_{k}$ a function of $\varepsilon_{t-k}$, the impulse responses to a given structural shock depend on the value of that shock at the time of shock. For instance, a positive shock may trigger a different impulse responses than a negative shock. With $\boldsymbol{\Psi}_{k}$ a function of $z_{t-k}$, the impulse responses to a structural shock depend on the value of $z$ at the time of that shock. For instance, the impulse responses may be different depending on the state of the business cycle (e.g., the level

\footnotetext{
${ }^{7}$ As we argue in two paragraphs, a VAR is ill-suited to capture such non-linearities.
} 
of unemployment) at the time of the shock.

Importantly, our starting point is not a structural Vector AutoRegression (VAR). While the use of a VAR is a common way to estimate a moving-average model, it relies on the existence of a VAR representation. However, in a non-linear world where $\boldsymbol{\Psi}_{k}$ depends on the sign of the shocks $\varepsilon$ as in (1), the existence of a VAR is compromised, because inverting (1) is difficult. Thus, in this paper, we work with an empirical method that side-steps the VAR and instead directly estimates the vector moving average model (1). We do so by means of Gaussian Mixture Approximations of the impulse responses that we describe next.

\subsection{Gaussian Mixture Approximations (GMA) of impulse responses}

Estimating moving-average model is notoriously difficult, because the number of free parameters $\boldsymbol{\Psi}_{k}$ in (1) is very large or infinite. To address this issue, our strategy consists in parameterizing the impulse response functions, and more precisely in using Gaussian basis functions to approximate impulse response functions.

Since the intuition and benefits of our approach can be understood in a linear context, this section introduces the Gaussian Mixture Approximation (GMA) of impulse responses in a linear context, i.e., where $\mathbf{\Psi}_{k}\left(\varepsilon_{t-k}, z_{t-k}\right)=\mathbf{\Psi}_{k}$. We postpone non-linear models to the next sections.

Denote $\psi(k)$ the representative element of matrix $\mathbf{\Psi}_{k}$, so that $\psi(k)$ is the value of the impulse response function $\psi$ at horizon $k$. A Gaussian Mixture Approximation of $\psi$ consists in decomposing $\psi$ into a sum of Gaussian basis functions, i.e., positing

$$
\psi(k)=\sum_{n=1}^{N} a_{n} e^{-\left(\frac{k-b_{n}}{c_{n}}\right)^{2}-\left(\frac{k-b_{n}}{c_{n}}\right)^{2}}, \quad \forall k>0
$$

with $a_{n}, b_{n}$, and $c_{n}$ parameters to be estimated. Since model (2) uses $N$ Gaussian basis functions, we refer to this model as a GMA of order $N$, or $G M A(N) .{ }^{8}$

\footnotetext{
${ }^{8}$ The GMA parametrization of $\psi$ may or may not include the contemporaneous impact coefficient, that is one may choose to use the approximation (18) for $k>0$ or for $k \geq 0$. In this paper, we treat $\psi(0)$ as a free
} 
The advantage of a GMA, and its use for studying the (possibly non-linear) effects of shocks, rest on the fact that, in practice, only a very small number of Gaussian basis functions are needed to approximate a typical impulse response function, allowing for efficiency gains and opening the door to estimating non-linearities.

Intuitively, impulse response functions of variables are often found to be monotonic or hump-shaped. In such cases, one or two Gaussian functions can already provide a very good approximate description of the impulse response. To illustrate this observation, Figure 1 plots the impulse response functions of government spending, taxes and output to a shock to government spending estimated from a standard VAR specification with a recursive ordering, ${ }^{9}$ along with the corresponding $G M A(1)$, the Gaussian approximations with only one Gaussian function, i.e., using the approximation

$$
\psi(k) \simeq a e^{-\frac{(k-b)^{2}}{c^{2}}}, \quad \forall k>0
$$

We can see that a one-Gaussian parametrization -a $G M A(1)$ - already does a good job at capturing the impulse responses implied by the VAR. With a $G M A(2)$, the impulse responses are very close to those of the VAR (Figure 1). For illustration, Figure 2 plots the Gaussian basis functions used for each impulse response in the GMA(2) case.

The small number of free parameters (only three per impulse response function in the one-Gaussian case), has two important advantages. First, it allows us to directly estimate the impulse response functions from the MA representation (1). ${ }^{10}$ Second, it will allow us to later add more degrees of freedom and allow for asymmetric or non-linear effects of shocks to government spending.

parameter for additional flexibility.

${ }^{9}$ We describe the exact specification in the next section.

${ }^{10}$ For instance, with 4 variables, we only have $3 * 4^{2}=48$ parameters (ignoring intercepts) to estimate to capture the whole set of impulse response functions $\left\{\boldsymbol{\Psi}_{k}\right\}_{k=1}^{\infty}$. In comparison, a corresponding VAR with 4 lags has 64 parameters. 


\subsection{The structural identifying assumptions}

Models like (1) are under-identified without additional restrictions. To identify government spending shocks, the fiscal policy literature has mainly followed two approaches: ${ }^{11}$ (i) a recursive identification scheme, and (ii) a narrative identification scheme. In this paper, we will consider both alternatives. This has two main advantages. First, it will allow us to assess the robustness of our findings across identification schemes. Second, it will allows us to relate to the current debate on the size of the multiplier and to better understand the wide range of estimates in the literature. ${ }^{12}$

\subsubsection{Identification from a recursive ordering}

The first identification scheme was proposed by Blanchard and Perotti (2002) and consists of a short-run restriction, i.e., a restriction on $\mathbf{\Psi}_{0}$, the matrix capturing the contemporaneous impact of a shock. Government spending is assumed to react with a lag to shocks affecting macro variables, so that in a system where $\boldsymbol{y}_{t}$ includes government spending, taxes and output, government spending is ordered first and $\boldsymbol{\Psi}_{0}$ has its first row filled with 0 except for the diagonal coefficient. This identification scheme was recently challenged because of anticipation effects (Ramey, 2011), as some innovations to government spending were found to be anticipated by agents. We thus follow Auerbach and Gorodnichenko (2012), who addressed the anticipation issue by augmenting the vector $\boldsymbol{y}_{t}$ with a professional forecast of the growth rate of government spending in order to soak up the forecastable components of shocks to government spending.

We now briefly describe how to estimate a multivariate GMA(N) model with a shortrun restriction using Bayesian methods. More details are available in the Appendix and in

\footnotetext{
${ }^{11}$ See e.g., Perotti (2008) and Ramey (2011, 2012) for overviews of the main identification schemes used in the literature.

${ }^{12}$ There is a third, methodological, advantage to considering both identification schemes. While Barnichon and Matthes (2016) discuss the use of GMAs in a multivariate model where both the shocks and the impulse responses are recovered simultaneously, they do not discuss univariate GMA models where shocks have been previously identified (as with the narrative approach). That latter class of models (whose range of application extends beyond that of fiscal shocks) is interesting because (i) estimation is very fast and (ii) with an independently identified shock series, it is possible to obtain a good initial guess of the magnitudes of the non-linearities.
} 
Barnichon and Matthes (2016). The key to estimating a moving-average model (1) is the construction of the likelihood function $p\left(\boldsymbol{y}^{T} \mid \boldsymbol{\theta}\right)$ of a sample of size $T$ for a moving-average model with parameter vector $\boldsymbol{\theta}$ and where a variable with a superscript denotes the sample of that variable up to the date in the superscript.

We use the prediction error decomposition to break up the density $p\left(\boldsymbol{y}^{T} \mid \boldsymbol{\theta}\right)$ as follows: ${ }^{13}$

$$
p\left(\boldsymbol{y}^{T} \mid \boldsymbol{\theta}\right)=\prod_{t=1}^{T} p\left(\boldsymbol{y}_{t} \mid \boldsymbol{\theta}, \boldsymbol{y}^{t-1}\right)
$$

Then, to calculate the one-step-ahead conditional likelihood function $p\left(\boldsymbol{y}_{t} \mid \boldsymbol{\theta}, \boldsymbol{y}^{t-1}\right)$, we assume that all innovations $\left\{\varepsilon_{t}\right\}$ are Gaussian with mean zero and variance one, ${ }^{14}$ and we note that the density $p\left(\boldsymbol{y}_{t} \mid \boldsymbol{y}^{t-1}, \boldsymbol{\theta}\right)$ can be re-written as $p\left(\boldsymbol{y}_{t} \mid \boldsymbol{\theta}, \boldsymbol{y}^{t-1}\right)=p\left(\boldsymbol{\Psi}_{0} \varepsilon_{t} \mid \boldsymbol{\theta}, \boldsymbol{y}^{t-1}\right)$ since

$$
\boldsymbol{y}_{t}=\boldsymbol{\Psi}_{0} \varepsilon_{t}+\sum_{k=1}^{K} \boldsymbol{\Psi}_{k} \varepsilon_{t-k}
$$

Since the contemporaneous impact matrix is a constant, $p\left(\boldsymbol{\Psi}_{0} \varepsilon_{t} \mid \boldsymbol{\theta}, \boldsymbol{y}^{t-1}\right)$ is a straightforward function of the density of $\varepsilon_{t}$.

To recursively construct $\varepsilon_{t}$ as a function of $\boldsymbol{\theta}$ and $\boldsymbol{y}^{t}$, we need to uniquely pin down the value of the components of $\varepsilon_{t}$, that is we need that $\boldsymbol{\Psi}_{0}$ is invertible. We impose this restriction by only keeping parameter draws for which $\boldsymbol{\Psi}_{0}$ is invertible. It is also at this stage that we impose the identifying restriction. We order variables in $\boldsymbol{y}$ such that the professional forecast of the growth rate of government spending enters first and government spending enters second. Then, our identifying restriction is that $\boldsymbol{\Psi}_{0}$ has its first two rows filled with 0 except for the diagonal coefficients. Finally, to initialize the recursion, we set the first $K$ values of $\varepsilon$ to zero. $^{15,16}$

\footnotetext{
${ }^{13}$ To derive the conditional densities in decomposition (4), our parameter vector $\boldsymbol{\theta}$ thus implicitly also includes the $K$ initial values of the shocks: $\left\{\varepsilon_{-K} \ldots \varepsilon_{0}\right\}$. We will keep those fixed throughout the estimation and discuss alternative initializations below.

${ }^{14}$ The estimation could easily be generalized to allow for non-normal innovations such as t-distributed errors.

${ }^{15}$ Alternatively, we could use the first $K$ values of the shocks recovered from a structural VAR.

${ }^{16}$ When $K$, the lag length of the moving average (1), is infinite, we truncate the model at some horizon $K$, large enough to ensure that the lag matrix coefficients $\boldsymbol{\Psi}_{K}$ are "close" to zero. Such a $K$ exists since the
} 
To explore the posterior density, we use a Metropolis-within-Gibbs algorithm (Robert and Casella, 2004) with the blocks given by the different groups of parameters in our model; $a, b$, and $c$. The elicitation of priors is described in the Appendix.

\subsubsection{Identification from a narrative approach}

The second main identification scheme is based on a narrative approach and was proposed by Ramey (2011), building on Ramey and Shapiro (1998).

In Ramey and Shapiro (1998), wars provide exogenous variations in government spending, because the entries into war (such as World-War II or the Korean war) (i) were exogenous to domestic economic developments, and (ii) led to large increases in defense spending. Generalizing this idea, Ramey (2011) identifies unexpected changes in anticipated future defense expenditures by using news sources to measure expectations and expectation surprises.

Incorporating narrative identification shocks into a GMA model is relatively straightforward. Indeed, in that case, it is no longer necessary to specify a self-contained model capturing the relevant features of the economy (and thus a multivariate moving average model), and one can directly estimate a univariate model -a univariate GMA- capturing the impulse response of any variable of interest to the independently identified structural shocks.

Taking $y_{t}$ to be one of the variables of $\boldsymbol{y}_{t}$, (1) implies that

$$
y_{t}=\sum_{k=0}^{K} \psi(k) \varepsilon_{t-k}^{G}+u_{t}^{y}
$$

with $\left\{\varepsilon_{t}^{G}\right\}$ the (narratively-identified) shocks to government spending, $\psi($.$) the impulse re-$ sponse function of $y$ to shock $\varepsilon^{G}$, and $u_{t}^{y}$ the residual. ${ }^{17}$ By using a Gaussian Mixture Approxvariables are stationary.

${ }^{17}$ The residual satisfies $u_{t}=\sum_{j} \sum_{k=0}^{K} \psi_{j}(k) \varepsilon_{t-k}^{(j)}$ where $\left\{\varepsilon_{t}^{(j)}\right\}$ are the other $j$ shocks affecting the economy and $\psi_{j}($.$) captures the impulse response function to shock \varepsilon_{t}^{(j)}$. 
imation of $\psi($.$) :$

$$
\psi(k)=\sum_{n=1}^{N} a_{n} e^{-\left(\frac{k-b_{n}}{c_{n}}\right)^{2}}, \quad \forall k>0
$$

we can estimate (6) in two ways: (i) with Bayesian methods assuming normally distributed residuals $\left\{u_{t}\right\}$ to construct the likelihood or (ii) by minimizing the sum of squared residuals (since the problem is relatively low dimensional). In practice, we will use a likelihood criteria to be consistent with our Bayesian approach, but we will use the estimates from a sum-of-squared residuals minimization (which has the advantage of not requiring a distribution assumption for the residuals $\left\{u_{t}\right\}$ ) as our initial guess. In addition, since the $u_{t} \mathrm{~s}$ are serially correlated by construction, in order to improve efficiency, we will allow for serial correlation in $u_{t}$ by positing that $u_{t}$ follows an autoregressive process. More details are provided in the Appendix.

\subsection{Calculating government spending multipliers}

We define the government spending multiplier as in the literature, and we consider two definitions: (i) the "max" multiplier

$$
m_{\max }=\max _{k \in[0, K]} \psi_{Y}(k) / \max _{k \in[0, K]} \psi_{G}(k)
$$

and (ii) the "sum" multiplier

$$
m_{\text {sum }}=\sum_{k=0}^{K} \psi_{Y}(k) / \sum_{k=0}^{K} \psi_{G}(k)
$$

where $\psi_{Y}($.$) and \psi_{G}($.$) denote respectively the impulse response function of output (denoted$ $Y$ from now on) and government spending (denoted $G$ from now on) to a spending shock.

Since the multiplier captures a ratio of changes in the levels of $Y$ and $G$, while the impulse responses are estimated for variables in logs, we need to convert the estimated impulse responses into dollar units. While a standard approach in the literature is to use an ex-post conversion

based on the approximation $\frac{d Y}{d G} \simeq \frac{d \ln Y}{d \ln G} \bar{Y}$ where $\frac{\bar{Y}}{G}$ is the sample average of the GDP to 
government spending ratio, Ramey, and Zubairy (2014) argue that this approach can lead to biased multiplier estimates, because $\frac{Y}{G}$ can display large movements over the sample period. We thus use instead an ex-ante conversion approach as in Gordon and Krenn (2010) and Ramey (2016), and before estimation we re-scale all variables by "potential output", where potential output $\left(Y^{\text {pot }}\right)$ is estimated from a quadratic trend.

While obtaining the posterior distribution of the multiplier is straightforward in a multivariate GMA where we jointly estimate the impulse responses of $Y$ and $G$ (as in the recursive identification scheme), the posterior distribution of the multiplier cannot be obtained from the separate univariate models of $Y$ and $G$ (as in the narrative identification scheme). To address this issue, while at the same time improving the efficiency of our estimation procedure, we thus estimate a SUR-type (Seemingly Unrelated Regression) model by jointly estimating the impulse responses of $y=Y / Y^{p o t}$ and $g=G / Y^{\text {pot }}$ to news to defense spending. Specifically, we estimate the model

$$
\left(\begin{array}{l}
y_{t} \\
g_{t}
\end{array}\right)=\sum_{k=0}^{K}\left(\begin{array}{c}
\psi_{y}(k) \\
\psi_{g}(k)
\end{array}\right) \varepsilon_{t-k}^{G}+\left(\begin{array}{c}
u_{t}^{y} \\
u_{t}^{g}
\end{array}\right)
$$

where the vector $\mathbf{u}_{t}=\left(\begin{array}{c}u_{t}^{y} \\ u_{t}^{g}\end{array}\right)$ follows a VAR process with

$$
\mathbf{u}_{t}=\mathbf{\Upsilon}(\mathbf{L}) \mathbf{u}_{t-1}+\boldsymbol{\eta}_{t}
$$

with $\boldsymbol{\Sigma}=E \boldsymbol{\eta}_{t} \boldsymbol{\eta}_{t}^{\prime}$ and $\boldsymbol{\Upsilon}(\mathbf{L})$ matrices to be estimated. As in the univariate GMA case, the likelihood can be constructed from the prediction error decomposition by assuming that $\boldsymbol{\eta}_{t}$ is i.i.d. and follows a multivariate normal distribution. More details are provided in the Appendix.

From the estimated posterior distribution of the parameters, it is straightforward to obtain the posterior distribution of any function of $\psi_{y}$ and $\psi_{g}$, and thus to obtain the posterior 
distribution of the multiplier.

\section{The asymmetric government spending multiplier}

We now turn to studying the (possibly) asymmetric effects of government spending shocks. We first describe how to introduce asymmetry in a GMA model, and then present the estimation results using (i) a recursive identification scheme à la Auerbach and Gorodnichenko (2012), and (ii) a narrative identification scheme à la Ramey (2011). We leave a detail description of the estimation of such models (which is a simple extension of the linear case described above) for the appendix.

\subsection{Introducing asymmetry}

To allow for asymmetry, we let $\boldsymbol{\Psi}_{k}$ depend on the sign of the government spending shock $\varepsilon^{G}$, i.e., we let $\boldsymbol{\Psi}_{k}$ take two possible values: $\boldsymbol{\Psi}_{k}^{+}$or $\boldsymbol{\Psi}_{k}^{-}$. Specifically, a general model that allows for asymmetric effects of shocks would write

$$
\boldsymbol{y}_{t}=\sum_{k=0}^{\infty}\left[\boldsymbol{\Psi}_{k}^{+} 1_{\varepsilon_{\ell, t-k}>0}+\boldsymbol{\Psi}_{k}^{-} 1_{\varepsilon_{\ell, t-k}<0}\right] \varepsilon_{t-k}
$$

with $\boldsymbol{\Psi}_{k}^{+}$and $\boldsymbol{\Psi}_{k}^{-}$the lag matrices of coefficients for, respectively, positive and negative government spending shocks.

Denoting $\psi_{i}^{G+}(k)$, the impulse response of variable $i$ at horizon $k$ to a positive government spending shock and similarly for $\psi_{i}^{G-}(k)$, a GMA(N) model of the impulse response function $\psi_{i}^{G+}$ would write

$$
\psi_{i}^{G+}(k)=\sum_{n=1}^{N} a_{i, n}^{+} e^{-\left(\frac{k-b_{i, n}^{+}}{c_{i, n}^{+}}\right)^{2}}, \forall k>0
$$

with $a_{i, n}^{+}, b_{i, n}^{+}, c_{i, n}^{+}$some constants to be estimated. A similar expression would hold for $\psi_{i}^{G-}(k)$. 


\subsection{Results from a recursive identification scheme}

To identify innovations to government spending, we first follow Auerbach and Gorodnichenko (2012), and we consider the vector $\left(\Delta g_{t \mid t-1}^{F}, g_{t}, \tau_{t}, y_{t}\right)^{\prime}$, where $g$ is log real government (federal, state, and local) purchases (consumption and investment), $\tau$ is log real government receipts of direct and indirect taxes net of transfers to businesses and individuals, and $y$ is log real gross domestic product (GDP) in chained 2000 dollars, and $\Delta g_{t \mid t-1}^{F}$ is the growth rate of government spending at time $t$ forecasted at time $t-1$. As described in Section 2, we include the anticipated growth rate of government spending in order to soak up any forecastable changes in government spending. For $\Delta g_{t \mid t-1}^{F}$, we combine Greenbook and SPF quarterly forecasts following Auerbach and Gorodnichenko (2012) and extending their dataset so that our sample cover 1966q1-2014q4.

Figure 3 plots the impulse responses estimated using a VAR with 4 lags (dashed black line) and a GMA(2), i.e., using 2 Gaussian basis functions (thick line) where we allow for a linear trend for each variable. ${ }^{18}$ The error bands cover 90 percent of the posterior probability. The upper panel plots the impulse responses to a positive shock to $G$, while the lower panel plots the impulse responses to a negative shock to G.

When comparing impulse responses to positive and negative shocks, it is important to keep in mind that the impulse responses to negative shocks were multiplied by -1 in order to ease comparison across impulse responses. With this convention, when there is no asymmetry, the impulse responses are identical in the top panel (responses to a positive shock) and in the bottom panels (responses to a negative shock). Finally, the magnitude of the fiscal shock is chosen to generate a peak effect on government spending of 1 in order to facilitate the interpretation of the results.

The results show that the impulses responses are strongly asymmetric: ${ }^{19}$

\footnotetext{
${ }^{18}$ The loose priors for the GMA parameters are detailed in the Appendix. To determine the appropriate number of Gaussian functions, i.e., the order $\mathrm{N}$ of the GMA, we use posterior odds ratios to compare models with increasing number of mixtures. We select the model with the highest posterior odds ratio. This approach can be seen as analogous to the choice of the number of lags in VAR models using Bayesian Information Criteria.

${ }^{19}$ Another way to evaluate the significance of our results is to use Bayesian model comparison. The asymmetric GMA displays marginal data densities that are substantially larger than the marginal data density of a corresponding Bayesian VAR with loose, but proper, Normal-Whishart priors.
} 
Starting with the left panels, the thick blue line depicts the response of $\mathrm{G}$ to a positive spending shock (an expansionary shock), while the red line depicts the shock to a negative spending shock (a contractionary shock). The responses to positive and negative shocks are similar although the response to a negative shock appears slightly more persistent.

We now turn to the impulse response of output, where, thanks to our ex-ante conversion, the peak of the impulse response can be directly read as the "max" government spending multiplier. We can see that the response of output is strong following a contractionary G shock but is not significantly different from zero following an expansionary G shock.

The strong asymmetric responses of Y imply strong asymmetries in the spending multiplier. As shown in Table 1, the "max" multiplier is 1.42 for a contractionary shock but is only 0.12 for an expansionary shock. The asymmetry is also present if we consider the "sum" multiplier (calculated over the first 20 quarters). Table 1 shows that the "sum" multiplier to a spending contraction is 1.25 (taking a horizon of 20 quarters), while the expansionary multiplier is -0.15 .

Figure 3 shows that the response of taxes is not behind the asymmetric size of the multiplier. While the response of taxes is not different from zero following an expansionary shock, taxes declined markedly following a contractionary shock (recall that the impulse responses to contractionary shocks are multiplied by -1 ). Thus, the tax response should make the adverse effect of contractionary fiscal policy on output smaller, not larger.

\subsection{Results from a narrative identification scheme}

We now turn to Ramey's narrative identification scheme, and we explore the asymmetry of the multiplier following unexpected changes in anticipated future defense expenditures. We estimate impulse response functions from a scalar GMA model with 2 Gaussian basis functions using quarterly data over $1939-2014 .{ }^{20}$

Figure 4 plots the impulse responses of government spending and output to news shocks, and Table 1 reports the corresponding sizes of the multiplier.

\footnotetext{
${ }^{20}$ The loose priors for the GMA parameters are detailed in the Appendix. The number of Gaussian basis functions was determined by model comparison using Bayes factors.
} 
The multipliers estimated from a linear GMA model are significantly lower than 1, with a point estimate of 0.72 for the "max" multiplier and 0.73 for the "sum" multiplier. But again, the picture changes when we allow for asymmetry, and the impulse responses and the size of the multiplier differ markedly between expansionary and contractionary shocks. The multiplier following a positive news shock is lower than 1 , at respectively 0.49 and 0.51 for the "max" and "sum" multipliers. In contrast, the multiplier following a negative shock is above one, with the "max" multiplier at 1.03 and the "sum" multiplier at 1.76.

Importantly, for both multipliers, the negative multiplier is significantly larger than the negative multiplier. Thus, these results confirm our previous findings based on a recursive identification scheme: the government multiplier is different for positive and negative shocks to public spending, with the multiplier consistently significantly below 1 when government spending increases but above one when government spending decreases.

\subsection{Digging deeper: the behavior of investment, consumption and interest rate}

To dig deeper into the asymmetric response of the economy to government spending shocks, we now study the impulse responses of different macro variables. As always, we present results for the two identification schemes.

Figures 5 and 6 plot the impulse responses of investment (I), consumption (C) and the fed funds rate (FFR) to positive and negative government spending shocks identified respectively with a recursive scheme and a narrative approach. In addition, Figure 6 also reports the response of the average marginal tax rate compiled by Barro and Redlick (2011). To obtain these impulse responses, we estimate a univariate-GMA like equation (6) with the shock series $\left\{\varepsilon_{t}^{G}\right\}$ given either by Ramey news shocks series or by the series of shocks identified from the multivariate GMA with a recursive ordering. ${ }^{21}$

\footnotetext{
${ }^{21}$ In the case of recursively identified shocks, the $\left\{\varepsilon_{t}^{G}\right\}$ shocks used in (6) are the shocks recovered from the asymmetric vector-GMA. More specifically, the Bayesian estimation of the vector-GMA model (10) delivers a posterior distribution of the $\left\{\varepsilon_{t}^{G}\right\}$ shocks, which can then be used in the univariate-GMA (6) to obtain a posterior distribution of the impulse response function of $\mathrm{C}$, I or FFR.
} 
When comparing impulse responses to positive and negative shocks, it is important to keep in mind that the impulse responses to negative shocks were multiplied by -1 in order to ease comparison across impulse responses. With this convention, when there is no asymmetry, the impulse responses are identical in the left-hand panels (responses to a positive shock) and in the right-hand panels (responses to a negative shock).

The main result that emerges across both identification schemes is that the asymmetric size of the multiplier is related to the very asymmetric impulse response of investment. In fact, investment significantly declines following a government spending shock, no matter the sign of the shock: investment is crowded-out following an expansionary spending shock, and it is not crowded-in following a contractionary spending shock. In contrast, the asymmetry in the response of consumption is not as striking, and the estimates are uncertain as reflected by the large posterior bands.

Turning to the response of the fed funds rate, we can see that, in both identification schemes, the interest rate moves in opposite direction to government spending, suggesting that the interest rate response amplifies the response of output to the spending shock. Although the error bands are too large to be conclusive in the narrative approach, the response of the fed funds rate is slightly stronger following a contractionary shock, which could explain some of the asymmetry in the response of output and investment.

Finally, the last row of Figure 6 plots the response of the average marginal tax rate to a Ramey news shock. We can see that the tax rate response displays little asymmetry, although the response is somewhat larger following an expansionary shock. This picture is in contrast with the tax response to a recursively-identified shock, where taxes did not respond following an expansionary shock. The fact that the asymmetry in the size of the multiplier can be present across different responses of the tax rate suggests that the method of financing is not behind the asymmetry in the size of the multiplier. 


\subsection{Robustness Check: Evidence of Asymmetry from Local Projections}

Since our approach relies on the parametrization of the impulse response functions with Gaussian basis functions, in this section, we examine the robustness of our previous results to this parametrization. The idea of the robustness check is to not rely on a GMA but instead to use a non-parametric method -Jorda's (2005) Local Projections (LP)-, which imposes little structure on the data generating process (DGP) and is thus more robust to mis-specification. The main drawback of such an LP-based approach is that (unlike GMA) it does not simultaneously recover the shocks and model parameters. Instead, a series of shocks must have been previously identified. In this robustness section, we thus only focus on the narrative approach and on the effects of the (independently identified) Ramey news shocks.

To first have a linear LP benchmark for the effects of news shocks to defense spending, we run linear Local Projections, i.e. we estimate $H$ equations

$$
y_{t+h}=\alpha_{h}+\beta_{h} \varepsilon_{t}^{G}+\gamma^{\prime} x_{t}+u_{t}^{h}, \quad h=0,1, \ldots, H
$$

where $y_{t+h}$ is the variable of interest, $x_{t}$ contains four lags of $y_{t}$ and $\varepsilon_{t}^{G}$ is the Ramey news shocks at time $t$. The estimates of the impulse response of $y$ at horizon $h$, is then given by $\beta_{h}$. We use an horizon of $H=20$ quarters (or 5 years).

To allow for asymmetric effects of credit shocks, we allow for sign-dependence in $\beta_{h}$, that is we estimate the $H$ equations

$$
y_{t+h}=\alpha_{h}+\beta_{h}^{+} \varepsilon_{t}^{G+}+\beta_{h}^{-} \varepsilon_{t}^{G-}+\gamma^{\prime} x_{t}+u_{t}^{h}, \quad h=0,1, \ldots, H
$$

where $\beta_{h}^{+}$is the response to a positive credit supply shock $\varepsilon_{t}^{G+}$, and $\beta_{h}^{-}$is the response to a negative credit supply shock $\varepsilon_{t}^{G-}$. We estimate (12) and (13) for the main variables considered so far, and Figure 7 shows the impulse response functions of government spending, output, investment and consumption.

Overall, the results are very similar to the results obtained with GMA models, although 
the error bands are considerably larger with Local Projections, which is not surprising given the lower efficiency of this approach (see e.g., Ramey, 2012). First, we again obtain that the multiplier is above 1 for a contractionary shock but is substantially below 1 for an expansionary shock. $^{22}$ Second, looking at the subcomponents of output, we find that both investment and consumption decline whenever there is a news to government spending, i.e., regardless of the sign of the shock to government spending. ${ }^{23}$ In other words, these estimates confirm our striking conclusion from GMA models that shocks to government spending decrease investment, regardless of the sign of the shock.

Overall, we conclude from this robustness exercise that a) nonlinearities in the effects of government spending shocks are important, and b) our previous conclusions on the asymmetric effects of shocks are not driven by the parametric restrictions imposed by Gaussian Mixture Approximations.

\section{The asymmetric and state-dependent government spending multiplier}

In an influential paper, Auerbach and Gorodnichenko (2012) find that the multiplier was much larger in recessions than in expansions, with a multiplier above 1.5 during recessions but below 0.5 during expansions. In contrast, Owyang, Ramey and Zubairy (2013) and Ramey and Zubairy (2014) find no evidence of state dependence when using Ramey's news shocks to government defense spending.

In this section, we explore whether the size of the multiplier depends on the state of the business cycle, and whether the magnitude of such state dependence depends on the sign of the government intervention. We first describe how we introduce state dependence into a

\footnotetext{
${ }^{22}$ The point estimates for the symmetric multipliers are 0.72 and 0.73 for respectively $m_{\max }$ and $m_{\text {sum }}$ (similarly to GMA estimates), while the point estimates for the asymmetric multipliers are only 0.65 and 0.63 for expansionary shocks but 1.18 and 1.70 for contractionary shocks.

${ }^{23}$ Recall that the impulse responses to negative shocks (right panels) were multiplied by -1 , so that if there were no asymmetry, the impulse responses should be identical in the left (response to a positive shock) and in the right panels (response to a negative shock).
} 
GMA model and then present the estimation results using both identification schemes: (i) the recursive identification scheme à la Auerbach and Gorodnichenko (2012), and (ii) the narrative identification scheme à la Ramey (2011). We leave a description of the estimation method (which is a simple extension of the asymmetric case above) for the appendix.

\subsection{Introducing asymmetry and state-dependence}

With asymmetry and state dependence in response to government spending shocks, the matrix $\boldsymbol{\Psi}_{k}^{+}$becomes $\boldsymbol{\Psi}_{k}^{+}\left(z_{t-k}\right)$, i.e., the impulse response to a positive shock depends on some indicator variable $z_{t}$ (and similarly for $\boldsymbol{\Psi}_{k}^{-}$).

To construct a model that allows for both asymmetry and state dependence, we build on the asymmetric GMA(N) model (11) and parametrize $\psi_{i}^{G+}$, the impulse response function of variable $i$ to a positive innovation to government spending, as

$$
\psi_{i}^{G+}(k)=\left(1+\gamma_{i}^{+} z_{t-k}\right) \sum_{n=1}^{N} a_{i, n}^{+} e^{-\left(\frac{k-b_{i, n}^{+}}{c_{i, n}^{+}}\right)^{2}}, \quad \forall k>0
$$

with $\gamma_{i}^{+}, a_{i, n}^{+}, b_{i, n}^{+}$and $c_{i, n}^{+}$parameters to be estimated. An identical functional form holds for $\psi_{i}^{G-}$.

In this model, the amplitude of the impulse response depends on the state of the business cycle (captured by the cyclical indicator $z_{t}$ ) at the time of the shock. In (14), the amplitude of the impulse response is a linear function of the indicator variable $z_{t}$. Such a specification will allow us to test whether a positive fiscal shock has a stronger effect on output in a recession than in an expansion.

Note that in specification (14), the state of the cycle is allowed to stretch/contract the impulse response, but the shape of the impulse response is fixed (because $a, b$ and $c$ are all independent of $z_{t}$ ). While one could allow for a more general model in which all variables $a$, $b$ and $c$ depend on the indicator variable, specification (14) has two advantages. First, with limited sample size, it will typically be necessary to impose some structure on the data, and 
imposing a constant shape for the impulse response is a natural starting point. Second, specification (14) generalizes trivially to GMAs of any order. The order of the GMA only determines the shape of the impulse response with higher order allowing for increasingly complex shapes. Then, for a given shape, the $\gamma$ coefficient can stretch or expand the impulse response depending on the state of the cycle.

\subsection{Results from a recursive identification scheme}

We estimate model (14), where we use as cyclical indicator $\left(z_{t}\right)$ the unemployment rate detrended by CBO's estimate of the natural rate (available from 1949 on). ${ }^{24}$

As a preliminary step, and to put our results into perspective, Figure 8 plots our cyclical indicator along with the identified government spending shocks implied by the posterior mode estimates. While the cyclical indicator has zero mean, it is right-skewed, a well-known property of the unemployment rate (e.g., Neftci, 1984). As a result, fiscal shocks are observed over values of (detrended) unemployment ranging mostly from -1 to 2 .

The first row of Figure 9 shows how the "max" multiplier depends on the state of the business cycle at the time of the shock. ${ }^{25}$ The left column reports the multiplier following positive (expansionary) shocks, and the right column reports the multiplier following negative (contractionary) shocks. The bottom panels of Figure 9 plot the histograms of the distributions of respectively contractionary shocks and expansionary shocks over the business cycle. This information is meant to get a sense of the range of (detrended) unemployment over which we identify the coefficients capturing state dependence.

We can once more see a stark asymmetry between positive and negative shocks. The multiplier associated with contractionary fiscal shocks depends strongly on the state of the cycle and reaches its highest value in times on high unemployment. For instance, the contractionary

\footnotetext{
${ }^{24}$ We detrend the unemployment rate to make sure that our results are not driven by slow moving trends (e.g., due to demographics) in the unemployment rate, which could make the unemployment rate a poor indicator of the amount of economic slack (see e.g. Barnichon and Mesters, 2015). Using the actual unemployment rate gives similar qualitative results for state dependence, but a posterior odds ratio calculation favors a model with detrended unemployment.

${ }^{25}$ Similar results hold for the "sum" multiplier.
} 
multiplier is about 1 around business cycle peaks but gets above to 2 around business cycle troughs. ${ }^{26}$ In contrast, the multiplier associated with expansionary fiscal shocks does not depend significantly or economically on the state of the cycle. It is small and not significantly different from 0 regardless of the level of unemployment.

\subsection{Results from a narrative identification scheme}

We now perform the same exercise but using the Ramey news shocks in the SUR-type GMA model with asymmetry and state dependence (model (9) with (14)) to allow the impulse responses to depend on both the sign of the news shock as well as the state of the cycle (captured by detrended unemployment) at the time of the shock. Since CBO's natural rate estimate is not available over 1939-2014 (the estimate only starts in 1949), we detrend unemployment with an HP-filter $\left(\lambda=10^{5}\right)$.

Figure 10 presents the results using the same formatting as Figure 9. Although the errorbands for the multiplier are larger than in Figure $9,{ }^{27}$ the results are strikingly similar to the ones obtained with the recursive identification: positive shocks have no state dependent effects on output, -the multiplier remaining around 0.5 regardless of the state of the cycle-, while negative shocks have stronger effects on output in recessions. And just like with the recursively identified shocks, the contractionary multiplier is about 1 around business cycle peaks but rises to about 2 around business cycle troughs.

\footnotetext{
${ }^{26}$ Interestingly, these results are consistent with the recent work of Caggiano et al. (2015), who find that state dependence in the size of the multiplier comes from extreme "events", and in particular deep recessions versus strong expansionary periods. This is what one would expect if the effect of the state of the cycle on the (contractionary) multiplier was close to linear: For small business cycle fluctuations, the size of the multiplier will not vary very much (Figure 9) and its variations will be hard to detect. It is only when the unemployment reaches high levels that the contractionary multiplier can reach values close to 2 and start differing markedly from its level during expansionary times.

${ }^{27}$ Large error-bands on negative news shocks are to be expected given the small number of negative news shocks in the Ramey sample.
} 


\section{Understanding the range of estimates in the literature}

The existence of asymmetry in the size of the multiplier helps reconcile seemingly contradictory findings in the literature on government spending multipliers. In particular, multipliers estimated from shocks to defense spending are often found to be smaller than multipliers estimated from a recursive identification scheme. A useful (if only approximate) characterization would be to say that narrative estimates generally imply multipliers less than one and not larger in recessions (Ramey and Zubairy, 2014), while VAR-based estimates imply multipliers close to or above one and larger in recessions (Auerbach and Gorodnichenko, 2012).

We now argue that these different results stem in part from variations in the sample of fiscal shocks used for identification in each method, and more specifically from variations in the relative importance of expansionary and contractionary shocks.

Figure 11 plots the distribution of Ramey news shocks along with the distribution of recursively-identified shocks (as in Auerbach and Gorodnichenko, 2012). Unlike with recursivelyidentified shocks whose distribution is (by construction) evenly distributed between positive and negative shocks, a few very large positive shocks dominate the sample of Ramey news shocks. ${ }^{28}$ As a result, the results from the narrative approach are more driven by positive shocks -unexpected news of increases in government spending--, and the estimated multiplier is small, because (according to our results) the multiplier associated with positive shocks is small. In contrast, because the spending shocks identified using a recursive identification scheme are more evenly distributed between positive and negative values, a recursive identification scheme will imply a larger multiplier than a narrative approach with an asymmetric shock distribution.

A similar reasoning can help rationalize the different estimates for state dependence. Studies based on Ramey's News shocks (Owyang, Ramey and Zubairy 2013, Ramey and Zubairy 2014) find little evidence for state dependence, because their results are more driven by posi-

\footnotetext{
${ }^{28}$ Indeed, it has been argued (e.g., AG) that the results of Ramey (2011) were driven by the large shocks of WWII and the Korean war.
} 
tive shocks, which (according to our results) display no state dependence. In contrast, studies such as Auerbach and Gorodnichenko (2012) based on a VAR find some evidence for state dependence, because (roughly) half of the identified shocks are negative shocks, which display strong state dependence.

An interesting implication of our findings lies in the interpretation of Auerbach and Gorodnichenko (2012)'s results. While Auerbach and Gorodnichenko (2012)'s findings have sometimes been interpreted as supporting the case for fiscal stimuli in recessions, our results caution against such a conclusion. We find no evidence that increases in government spending have larger multipliers during a recession (in fact, the multiplier is consistently below 1) and thus no support for stimulus programs in times of recession. However, we find that decreases in government spending during recessions have the largest multiplier, which suggests that austerity measures during recessions can be especially harmful.

\section{Conclusion}

This paper estimates the asymmetric effects of shocks to government spending by using Gaussian basis functions to approximate impulse response functions. Using either of the two main identification schemes in the literature -Blanchard-Perotti (2002) or Ramey (2011)-, we find that the multiplier is above 1 for contractionary shocks to government spending, but substantially below 1 for expansionary shocks. The spending multiplier is largest in recessions, as found in some earlier studies, but only because the multiplier for contractionary shocks is largest in recessions. The multiplier for expansionary shocks is always below 1 and not larger in recessions.

These results have two interesting policy implications. First, they strongly weaken the case for fiscal packages to stimulate the economy. Second, they caution that austerity measures may have a much higher output cost than suggested by linear estimates.

Treating expansionary and contractionary fiscal shocks separately also allows us to reconcile seemingly contradictory findings in the literature on government multipliers: While estimates 
from shocks to defense spending (Ramey, 2011, Barro and Redlick 2011) are generally less than 1, estimates from shocks recovered from VARs are often close to or above 1 and larger in recessions (Auerbach and Gorodnichenko, 2012). We argue that these different results stem in part from variations in the sample of fiscal shocks recovered by each method and more specifically from variations in the relative importance of expansionary and contractionary shocks.

We find that the asymmetry in the size of the multiplier is related to the strong asymmetric response of investment, with the results again consistent across the two different identification schemes: While expansionary spending shocks generate a significant drop in investment (i.e., crowd out investment), contractionary spending shocks also generate a significant drop in investment (i.e., do not crowd in investment). In other words, any shock to government spending appears to adversely affect private investment. An interesting corollary is that an increase in the variance of government spending shocks, i.e., an increase in the uncertainty associated with the path of government spending, would lower private investment. This would suggest that periods of high uncertainty in fiscal policy are detrimental for investment, a possibility that echoes a large literature focused on the adverse effects of uncertainty on the economy (Bloom, 2009, Baker, Bloom and Davis, 2015).

The strong asymmetry in the response of investment is also interesting in the context of an earlier empirical literature aimed at informing the debate on the most likely transmission mechanism of fiscal policy (Perotti, 2008, Ramey, 2011). While in most modern models the effects of fiscal policy occur through the consumption (and hours) response of households, our results indicate that the investment response may be a key channel in the transmission mechanism of fiscal policy, as also advocated for instance by Alesina, Ardagna, Perotti and Schiantarelli (2002).

Finally, an important goal for future research is to understand the mechanism generating the asymmetric effect of fiscal policy, with possible mechanisms involving downward wage 
rigidity in a model like Alesina, Ardagna, Perotti and Schiantarelli (2002) ${ }^{29}$ or occasionally binding constraints. ${ }^{30}$

\footnotetext{
${ }^{29}$ In Alesina, Ardagna, Perotti and Schiantarelli (2002), an increase in government spending can lead to wage pressures which hurt investment by the private sector. With downward wage rigidity, a decrease in government spending would not lower wages and thus would not stimulate investment.

${ }^{30}$ For instance, in a New-Keynesian model like Gali, Lopez-Salido and Valles (2007) where the hand-to-mouth behavior (and high MPC) of some agents could arise out of the presence of binding financial constraints, the size of the multiplier could depend on the sign of the spending shock. However, since we found that the asymmetry was strongest for the response of investment (and more uncertain for consumption), our results suggest that the financial constraint most relevant for the effect of fiscal policy would be at the firm level rather than at the household level.
} 


\section{Appendix A1: Bayesian estimation of multivariate GMA models}

In this section, we describe the implementation and estimation of multivariate GMA models where government spending shocks are identified from a recursive ordering as in described in the main text. We first describe how we construct the likelihood function by exploiting the prediction-error decomposition, discuss the estimation routine based on a multiple-block Metropolis-Hasting algorithm, prior elicitation, and finally determination of the order of the GMA. This section draws from Barnichon and Matthes (2016).

\section{Constructing the likelihood function}

We now describe how to construct the likelihood function $p\left(\boldsymbol{y}^{T} \mid \boldsymbol{\theta}, z^{T}\right)$ of a sample of size $T$ for the moving-average model (1) with parameter vector $\boldsymbol{\theta}$ and where a variable with a superscript denotes the sample of that variable up to the date in the superscript.

To start, we use the prediction error decomposition to break up the density $p\left(\boldsymbol{y}^{T} \mid \boldsymbol{\theta}\right)$ as follows: ${ }^{31}$

$$
p\left(\boldsymbol{y}^{T} \mid \boldsymbol{\theta}\right)=\prod_{t=1}^{T} p\left(\boldsymbol{y}_{t} \mid \boldsymbol{\theta}, \boldsymbol{y}^{t-1}\right) .
$$

To calculate the one-step-ahead conditional likelihood function needed for the prediction error decomposition, we assume that all innovations $\left\{\varepsilon_{t}\right\}$ are Gaussian with mean zero and variance one, and we note that the density $p\left(\boldsymbol{y}_{t} \mid \boldsymbol{y}^{t-1}, \boldsymbol{\theta}\right)$ can be re-written as $p\left(\boldsymbol{y}_{t} \mid \boldsymbol{\theta}, \boldsymbol{y}^{t-1}\right)=$ $p\left(\boldsymbol{\Psi}_{0} \varepsilon_{t} \mid \boldsymbol{\theta}, \boldsymbol{y}^{t-1}\right)$ since

$$
\boldsymbol{y}_{t}=\boldsymbol{\Psi}_{0} \varepsilon_{t}+\sum_{k=1}^{K} \boldsymbol{\Psi}_{k} \varepsilon_{t-k}
$$

Since the contemporaneous impact matrix $\Psi_{0}$ is a constant, $p\left(\Psi_{0} \varepsilon_{t} \mid \boldsymbol{\theta}, \boldsymbol{y}^{t-1}\right)$ is a straightforward function of the density of $\varepsilon_{t}$.

To recursively construct $\boldsymbol{\varepsilon}_{t}$ as a function of $\boldsymbol{\theta}$ and $\boldsymbol{y}^{t}$, we need to uniquely pin down the value of the components of $\varepsilon_{t}$ from (16), that is we need that $\boldsymbol{\Psi}_{0}$ is invertible. We impose this

\footnotetext{
${ }^{31}$ To derive the conditional densities in decomposition (15), our parameter vector $\boldsymbol{\theta}$ thus implicitly also includes the $K$ initial values of the shocks: $\left\{\varepsilon_{-K} \ldots \varepsilon_{0}\right\}$. We will keep those fixed throughout the estimation and discuss alternative initializations below.
} 
restriction by only keeping parameter draws for which $\boldsymbol{\Psi}_{0}$ is invertible. ${ }^{32}$ It is also at this stage that we impose the identifying restriction that $\boldsymbol{\Psi}_{0}$ has its first two rows filled with 0 except for the diagonal coefficients. Finally, to initialize the recursion, we set the first $K$ innovations $\left\{\varepsilon_{j}\right\}_{j=-K}^{0}$ to zero.

In the non-linear case where we have $\boldsymbol{\Psi}_{k}=\boldsymbol{\Psi}_{k}\left(\varepsilon_{t-k}, z_{t-k}\right)$, we proceed similarly. However, a complication arises when one allows $\mathbf{\Psi}_{0}$ to depend on the sign of the shock while also imposing identifying restrictions on $\mathbf{\Psi}_{0}$. The complication arises, because with asymmetry the system of equations implied by (16):

$$
\mathbf{\Psi}_{0}\left(\varepsilon_{t-k}, z_{t-k}\right) \varepsilon_{t}=\mathbf{u}_{t}
$$

where $\mathbf{u}_{t}=\boldsymbol{y}_{t}-\sum_{k=1}^{K} \boldsymbol{\Psi}_{k} \varepsilon_{t-k}$ need not have a unique solution vector $\varepsilon_{t}$, because $\boldsymbol{\Psi}_{0}\left(\varepsilon_{t}\right)$, the impact matrix, depends on the sign of the shocks, i.e., on the vector $\varepsilon_{t}$. However, in Barnichon and Matthes (2016), we show that this is not a problem (so that (17) has a unique solution vector $\left.\varepsilon_{t}\right)$ in a recursive identification scheme like the one considered in this paper.

Finally, when constructing the likelihood, to write down the one-step ahead forecast density $p\left(\boldsymbol{y}_{t} \mid \boldsymbol{\theta}, \boldsymbol{y}^{t-1}\right)$ as a function of past observations and model parameters, we use the standard result (see e.g., Casella-Berger, 2002) that for $\boldsymbol{\Psi}_{0}$ a function of $\varepsilon_{t}$ and $z_{t}$, we have

$$
p\left(\boldsymbol{\Psi}_{0}\left(\varepsilon_{t}, z_{t}\right) \varepsilon_{t} \mid \boldsymbol{\theta}, \boldsymbol{y}^{t-1}\right)=J_{t} p\left(\varepsilon_{t}\right)
$$

where $J_{t}$ is the Jacobian of the (one-to-one) mapping from $\varepsilon_{t}$ to $\boldsymbol{\Psi}_{0}\left(\varepsilon_{t}, z_{t}\right) \varepsilon_{t}$ and where $p\left(\varepsilon_{t}\right)$ is the density of $\varepsilon_{t} \cdot 33,34$

\footnotetext{
${ }^{32}$ Parameter restrictions (such as invertibility) are implemented by assigning a minus infinity value to the likelihood whenever the restrictions are not met.

${ }^{33}$ Recall that we assume that the indicator variable $z_{t}$ is a function of lagged values of $\boldsymbol{y}_{t}$ (so that $z_{t}$ is known conditional on $\boldsymbol{y}^{t-1}$ ) or that $z_{t}$ is a function of variables exogenous to $\boldsymbol{y}_{t}$ (and thus taken as given and known).

${ }^{34}$ In our case with asymmetry, this Jacobian is simple to calculate, but the mapping is not differentiable at $\varepsilon=0$. Since we will never exactly observe $\varepsilon=0$ in a finite sample, we can implicitly assume that in a small neighborhood around 0 , we replace the original mapping with a smooth function.
} 


\section{Estimation routine and initial guess}

To estimate our model, we use a Metropolis-within-Gibbs algorithm (Robert \& Casella 2004) with the blocks given by the different groups of parameters in our model (there is respectively one block for the $a$ parameters, one block for the $b$ parameters, one block for the $c$ parameters and one block for the constant and contemporaneous impact matrix $\Psi_{0}$ ).

To initialize the Metropolis-Hastings algorithm in an area of the parameter space that has substantial posterior probability, we follow a two-step procedure: first, we estimate a standard VAR using OLS on our data set, calculate the moving-average representation, and we use the impulse response functions implied by the VAR as our starting point. More specifically, we calculate the parameters of our GMA model to best fit the VAR-based impulse response functions. ${ }^{35}$ Second, we use these parameters as a starting point for a simplex maximization routine that then gives us a starting value for the Metropolis-Hastings algorithm.

In the non-linear models, we initialize the parameters capturing asymmetry and state dependence at zero (i.e., no non-linearity). This approach is consistent with the starting point (the null) of this paper: structural shocks have linear effects on the economy, and we are testing this null against the alternative that shocks have some non-linear effects. We then center the priors for these parameters at zero with loose priors, as described next.

\section{Prior elicitation}

We use (loose) Normal priors centered around the impulse response functions obtained from the benchmark (linear) VAR. Specifically, we put priors on the $a, b$ and $c$ coefficients that are centered on the values for $a, b$ and $c$ obtained by matching the impulse responses obtained from the VAR, as described in the previous paragraph. Specifically, denote $a_{i j, n}^{0}, b_{i j, n}^{0}$ and $c_{i j, n}^{0}$, $n \in\{1, N\}$ the values implied by fitting the GMA(N) to the VAR-based impulse response of variable $i$ to shock $j$. The priors for $a_{i j, n}, b_{i j, n}$ and $c_{i j, n}$ are centered on $a_{i j, n}^{0}, b_{i j, n}^{0}$ and $c_{i j, n}^{0}$,

\footnotetext{
${ }^{35}$ Specifically, we set the parameters of our model (the $a, b$ and $c$ coefficients) to minimize the discrepancy (sum of squared residuals) between the two sets of impulse responses.
} 
and the standard-deviations are set as follows $\sigma_{i j, a}=10, \sigma_{i j, b}=K$ and $\sigma_{i j, c}=K(K$ is the maximum horizon of the impulse response function). ${ }^{36}$ While there is clearly some arbitrariness in choosing the tightness of our priors, it is important to note that they are very loose and let us explore a large class of alternative specifications.

The use of informative priors is not critical for our approach, but we do this for a number of reason. First, since our current knowledge on the effect of government spending shocks is based to a large extent on VAR evidence, it seems natural (and consistent with the Bayesian approach) to impose priors centered on our current state of knowledge. Second, given the inherent difficulty in estimating moving-average models, the priors help discipline the estimation by keeping the parameters in a reasonable set of the parameter space. Finally, and while we could have used improper uniform prior, the use of proper priors allows us to compute posterior odds ratio, which are important to select the order of the moving-average and to compare different GMA models.

\section{Choosing $N$, the number of Gaussian basis functions}

To choose $N$, the order of the GMA model, we use posterior odds ratios (assigning equal probability to any two model) to compare models with increasing number of mixtures. We select the model with the highest posterior odds ratio.

\section{Appendix A2: Bayesian estimation of univariate and SUR-type GMA models}

The previous section described how to estimate multivariate GMA models when we simultaneously identify the structural shocks and estimate the impulse response functions. We now describe how to estimate models when the shocks have been previously identified (for instance,

\footnotetext{
${ }^{36}$ Note that these priors are very loose. This is easy to see for $a$ and $b$. For $c$, if it easy to show that $c \sqrt{\ln 2}$ is the half-life of the effect of a shock. Thus, $c=K$ corresponds to a very persistent impulse response function, since $K \sqrt{\ln 2}=38$ quarters.
} 
through a narrative approach as in Ramey, 2011). The model can be a univariate GMA model like (6) or a SUR-type GMA model like (9).

For ease of exposition, we focus on the univariate model first, since the SUR-type model is a simple extension of the univariate case. As with the multivariate GMA case, we use Bayesian methods and the key part is the construction of the likelihood. Recall from section 3 that we have a model of the form

$$
y_{t}=\sum_{k=0}^{K} \psi(k) \varepsilon_{t-k}^{G}+u_{t}
$$

with

$$
\psi(k)=\sum_{n=1}^{N} a_{n} e^{-\left(\frac{k-b_{n}}{c_{n}}\right)^{2}}
$$

where $a_{n}, b_{n}$ and $c_{n}$ can be functions of $\varepsilon_{t-k}^{G}$ (in the non-linear case), and where the residual is $u_{t} \equiv \sum_{j} \sum_{k=0}^{K} \psi_{j}(k) \varepsilon_{t-k}^{(j)}$.

Since $\left\{u_{t}\right\}$ is serially correlated by construction, in order to improve efficiency, we allow for serial correlation in $u_{t}$ by positing that $u_{t}$ follows an $A R(1)$ process. That is, we posit that $u_{t}=\rho u_{t-1}+\eta_{t}$ where $\eta_{t}$ is Normally distributed $N\left(0, \sigma_{\eta}^{2}\right)$ with $\sigma_{\eta}$ a parameter to be estimated. We set $\eta_{-1}$ and $\eta_{0}$ to zero, and from (18), it is straightforward to build the likelihood given a series of previously identified shocks $\left\{\varepsilon_{t}^{G}\right\}$. For prior elicitation, we proceed as with the multivariate GMA, and use very loose priors with $\sigma_{a}=10, \sigma_{b}=K$ and $\sigma_{c}=K$.

For a SUR-type model like (9), the estimation proceeds along the same lines as above, except that we take into account that the one-step forecast error $u_{t}$ is now a vector that follows a $\operatorname{VAR}(1)$ process instead of an $\operatorname{AR}(1)$ process.

Finally, to select $N$ (the number of Gaussian basis functions) in the initial guess, we use Bayes factors (as in the multivariate GMA) to determine the preferred order of the GMA.

\section{Estimation routine and initial guess}

As with the multivariate GMA, we use a Metropolis-within-Gibbs algorithm. Regarding the initial guess, an interesting advantage of a univariate GMA is that it is possible to compute a 
good initial guess, even in non-linear models.

\section{Obtaining a non-linear initial guess}

To obtain a good (possibly non-linear) initial guess in univariate and SUR-type GMA models, we proceed in two steps.

1. Recovering the $\left\{a_{n}\right\}$ factors given $\left\{b_{n}, c_{n}\right\}$

Assume that the parameters of the Gaussian kernels $-\left\{b_{n}, c_{n}\right\}_{n=1^{-}}^{N}$ are known, so that we have a "dictionary" of basis functions to decompose our impulse response. Then, estimating the coefficients $\left\{a_{n}\right\}_{n=1}^{N}$ in (18), a non-linear problem, can be recast into a linear problem that can estimated by OLS.

To see that, consider first a linear model where $\psi(k)$ is independent of $\varepsilon_{t-k}^{G}$. We then re-arrange (18) as follows:

$$
\begin{aligned}
\sum_{k=0}^{K} \psi(k) \varepsilon_{t-k}^{G} & =\sum_{k=0}^{K} \sum_{n=1}^{N} a_{n} e^{-\left(\frac{k-b_{n}}{c_{n}}\right)^{2}} \varepsilon_{t-k}^{G} \\
& =\sum_{n=1}^{N} a_{n} \sum_{k=0}^{K} e^{-\left(\frac{k-b_{n}}{c_{n}}\right)^{2}} \varepsilon_{t-k}^{G} .
\end{aligned}
$$

Defining

$$
X_{n, t}=\sum_{k=0}^{K} e^{-\left(\frac{k-b_{n}}{c_{n}}\right)^{2}} \varepsilon_{t-k}^{G},
$$

our estimation problem becomes a linear problem (conditional on knowing $\left\{b_{n}, c_{n}\right\}_{n=1}^{N}$ ):

$$
y_{t}=\sum_{n=1}^{N} a_{n} X_{n, t}+\alpha+\beta u_{t}
$$

where the $\left\{a_{n}\right\}$ parameters can be recovered instantaneously by OLS. Assuming that $u_{t}$ follows an $\operatorname{AR}(1)$, we can estimate the $\left\{a_{n}\right\}$ with a simple NLS procedure. 
The method described above in the linear case is straightforward to apply to a case with non-linearities. Consider for instance the case with asymmetry

$$
a_{n}\left(\varepsilon_{t-k}^{G}\right)=a_{n}^{+} 1_{\varepsilon^{G} \geq 0}+a_{n}^{-} 1_{\varepsilon^{G}<0} .
$$

Then, we can proceed as in the previous section and define the following right-hand side variables

$$
\left\{\begin{array}{l}
X_{n, t}^{+}=\sum_{k=0}^{K} h_{n}(k) \varepsilon_{t-k}^{G} 1_{\varepsilon \geq 0} \\
X_{n, t}^{-}=\sum_{k=0}^{K} h_{n}(k) \varepsilon_{t-k}^{G} 1_{\varepsilon<0}
\end{array}\right.
$$

and use OLS to recover $a_{n}^{+}$and $b_{n}^{-}$.

2. Choosing $\left\{b_{n}, c_{n}\right\}$

To estimate $\left\{b_{n}, c_{n}\right\}_{n=1}^{N}$ (and therefore $\left\{a_{n}\right\}_{n=1}^{N}$ from the OLS regression), we minimize the sum of squared residuals of (19) using a simplex algorithm (Matlab's fminsearch).

The advantages of this method are two folds: first, compared to a direct minimization of (19) that treats all three sets of parameters $a, b$ and $c$ as free parameters, our procedure is more efficient because it exploits the efficiency of OLS to recover $\left\{a_{n}\right\}$ given $\left\{b_{n}, c_{n}\right\}$. Second, compared to a likelihood-based method, the initial guess does not rely on the Normality of the innovations $\eta_{t}$. 


\section{References}

[1] Alesina, A., and S. Ardagna. "Large Changes in Fiscal Policy: Taxes versus Spending," In Tax Policy and the Economy, edited by Jeffrey R. Brown, vol. 24. Chicago: University of Chicago Press, pp. 35-68, 2010

[2] Alesina A, S Ardagna, R Perotti and F Schiantarelli, "Fiscal Policy, Profits, and Investment," American Economic Review, vol. 92(3), pages 571-589, 2002

[3] Almon, S. "The distributed lag between capital appropriations and expenditures," Econometrica, 33, January, 178-196, 1965

[4] Alspach D. and H. Sorenson H. "Recursive Bayesian Estimation Using Gaussian Sums," Automatica, Vol 7, pp 465-479, 1971

[5] Alspach D. and H. Sorenson H. "Nonlinear Bayesian Estimation Using Gaussian Sum Approximations," IEEE Transactions on Automatic Control, Vol 17-4, August 1972

[6] Auerbach A and Y Gorodnichenko. "Measuring the Output Responses to Fiscal Policy," American Economic Journal: Economic Policy, vol. 4(2), pages 1-27, 2012

[7] Auerbach A and Y Gorodnichenko. "Fiscal Multipliers in Recession and Expansion." In Fiscal Policy After the Financial Crisis, pp. 63-98, University of Chicago Press, 2013

[8] Baker S., N Bloom and S. Davis, "Measuring Economic Policy Uncertainty," CEP Discussion Papers dp1379, 2015

[9] Barnichon R. and C. Matthes. "Gaussian Mixture Approximations of Impulse Responses and the Non-Linear Effects of Monetary Shocks," Working Paper, 2016

[10] Barnichon R. and C. Matthes. "Imposing structural identifying restrictions in Gaussian Mixture Approximation (GMA) models," Working Paper, 2016 
[11] Barnichon R, and G. Mesters, "On the Demographic Adjustment of Unemployment," Working Paper, 2015

[12] Barro R. and C. Redlick. "Macroeconomic Effects From Government Purchases and Taxes," Quarterly Journal of Economics (2011) 126 (1): 51-102, 2011

[13] Ben Zeev, N. and E. Pappa, "Chronicle of a War Foretold: The Macroeconomic Effects of Anticipated Defense Spending Shocks," forthcoming Economic Journal, 2015

[14] Blanchard, O., and R. Perotti. "An Empirical Characterization of the Dynamic Effects of Changes in Government Spending and Taxes on Output." Quarterly Journal of Economics 117 (4): 1329-68, 2002

[15] Bloom, N. "The Impact of Uncertainty Shocks," Econometrica, vol. 77(3), pages 623-685, 05,2009

[16] Buhmann, M., Radial Basis Functions: Theory and Implementations, Cambridge University Press, 2003

[17] Caggiano, G., E. Castelnuovo, V., Colombo, and G. Nodari, "Estimating Fiscal Multipliers: News from a Nonlinear World," Economic Journal, 125(584), 746-776, 2015.

[18] Fisher, J., and R. Peters, "Using Stock Returns to Identify Government Spending Shocks," The Economic Journal, 120 (May 2010): 414-436.

[19] Gali, J., D. Lopez-Salido, and J. Valles, "Understanding the Effects of Government Spending on Consumption," Journal of the European Economic Association, 5: 227-270, 2007

[20] Gordon, R. and R. Krenn. "The End of the Great Depression: VAR Insight on the Roles of Monetary and Fiscal Policy," NBER Working Paper 16380, 2010.

[21] Guajardo, Jaime, Daniel Leigh, and Andrea Pescatori. "Expansionary Austerity: New International Evidence" IMF Working Paper 11/158, 2014 
[22] Hall, R. "By How Much Does GDP Rise If the Government Buys More Output?" Brookings Papers on Economic Activity, 2, 183-231, 2009

[23] Jorda O., "Estimation and Inference of Impulse Responses by Local Projections," American Economic Review, pages 161-182, March 2005

[24] Jorda O. and A. Taylor. "The Time for Austerity: Estimating the Average Treatment Effect of Fiscal Policy" Working Paper 2013-25 September 2013

[25] McLachlan, G. and D. Peel. Finite Mixture Models. Wiley Series in Probability and Statistics, 2000

[26] Mertens K. and M. Ravn. "Measuring the Impact of Fiscal Policy in the Face of Anticipation: A Structural VAR Approach," Economic Journal, Volume 120, Issue 544, pages 393-413, May 2010.

[27] Neftci, S. "Are Economic Time Series Asymmetric over the Business Cycle?," Journal of Political Economy, 92, 307-28, 1984.

[28] Owyang, M., V. Ramey and S. Zubairy. "Are Government Spending Multipliers Greater During Periods of Slack? Evidence from 20th Century Historical Data" American Economic Review Papers and Proceedings, May 2013

[29] Parker, J. "On Measuring the Effects of Fiscal Policy in Recessions," Journal of Economic Literature, American Economic Association, vol. 49(3), pages 703-18, September, 2011

[30] Perotti, R. "In Search of the Transmission Mechanism of Fiscal Policy," NBER Macroeconomics Annual, Volume 22, 2008

[31] Ramey, V. "Can Government Purchases Stimulate the Economy?," Journal of Economic Literature, vol. 49(3), pages 673-85, 2011

[32] Ramey V. "Identifying Government Spending Shocks: It's All in the Timing," Quarterly Journal of Economics 126, 1-50, 2011 
[33] Ramey, V. "Government Spending and Private Activity," NBER Chapters, in: Fiscal Policy after the Financial Crisis, pages 19-55, 2012

[34] Ramey V. "Comment on "Roads to Prosperity or Bridges to Nowhere? Theory and Evidence on the Impact of Public Infrastructure Investment", NBER Macroeconomics Annual, Volume 27, pages 147-153, 2012

[35] Ramey V. and S. Zubairy. "Government Spending Multipliers in Good Times and in Bad: Evidence from U.S. Historical Data ," Working Paper, 2014

[36] Ramey V. "Macroeconomic Shocks and Their Propagation," Working Paper, 2016.

[37] Riera-Crichton, D. C. Vegh, G. Vuletin, "Procyclical and countercyclical fiscal multipliers: Evidence from OECD countries," Journal of International Money and Finance, Volume 52, Pages 15-31, 2015

[38] Robert, C. and G. Casella Monte Carlo Statistical Methods Springer, 2004

[39] Walsh C. Monetary Theory and Policy, 3nd. ed., The MIT Press, 2010 

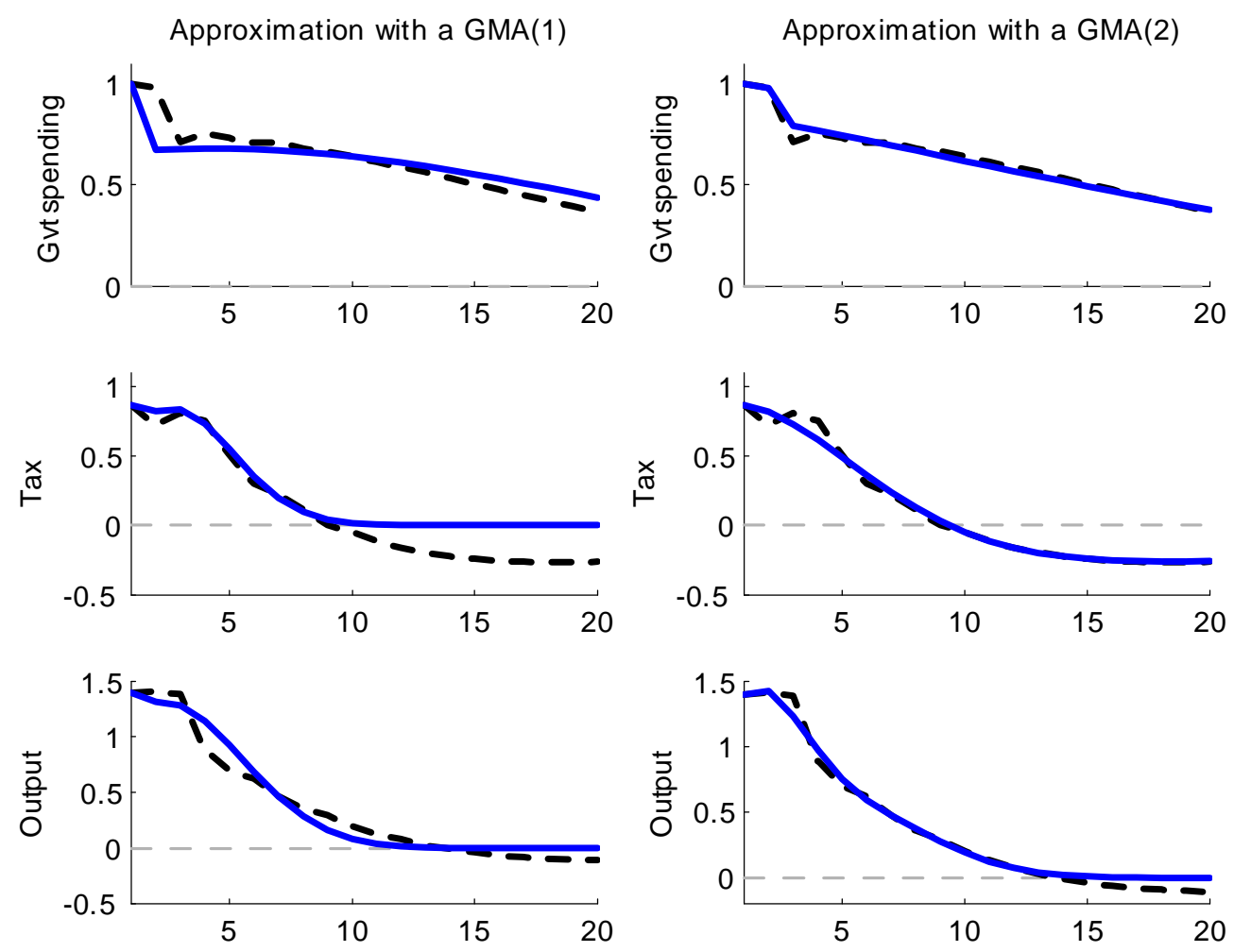

Figure 1: Impulse response functions (in percent) of government spending, government revenue ("Tax") and output to a one standard-deviation government spending shock. Impulse responses estimated with a VAR (dashed-line) or approximated using one Gaussian basis function (GMA(1), left-panel, thick line) or two Gaussian basis functions (GMA(2), right panel thick line). Estimation using quarterly data covering 1966-2014. 

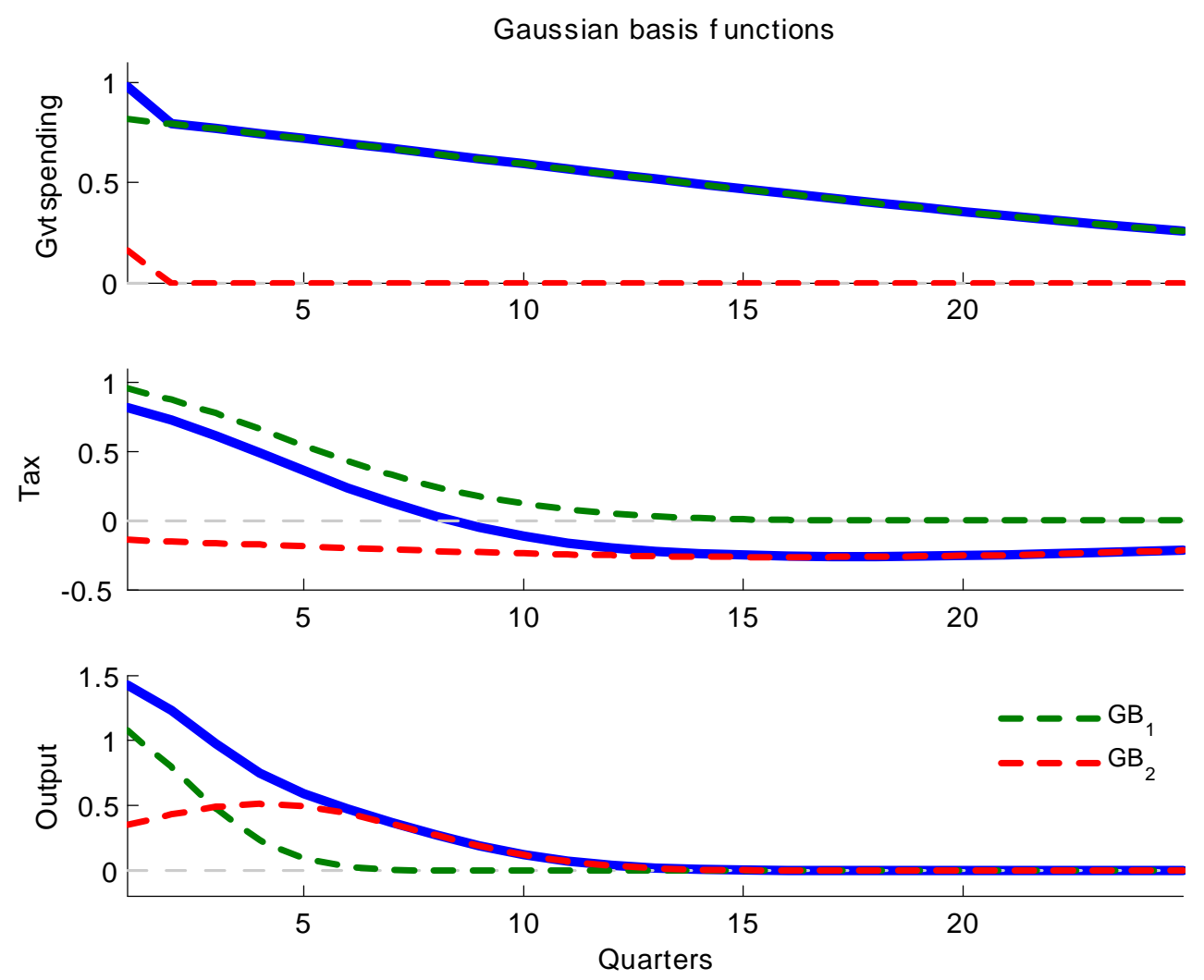

Figure 2: Gaussian basis functions (dashed lines) used by a GMA(2) to approximate the responses of unemployment, inflation and the fed funds rate to a monetary shock. The basis functions are appropriately weighted so that their sum gives the GMA(2) parametrization of the impulse response functions (solid lines) reported in the right-panels of Figure 1. 

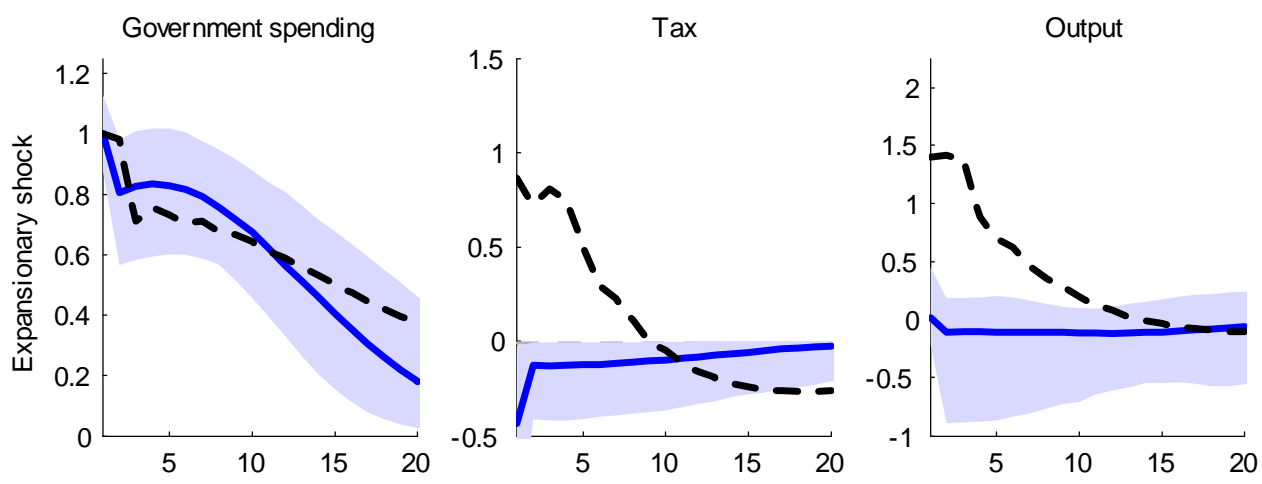

(-) Government spending

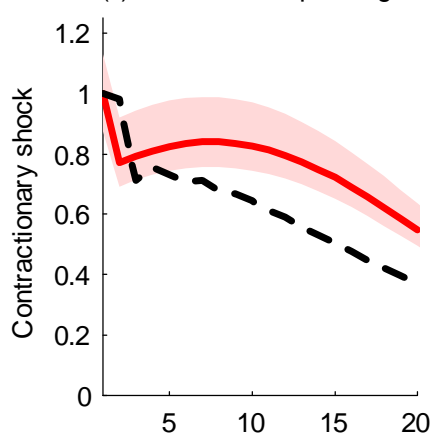

(-) Tax

(-) Output
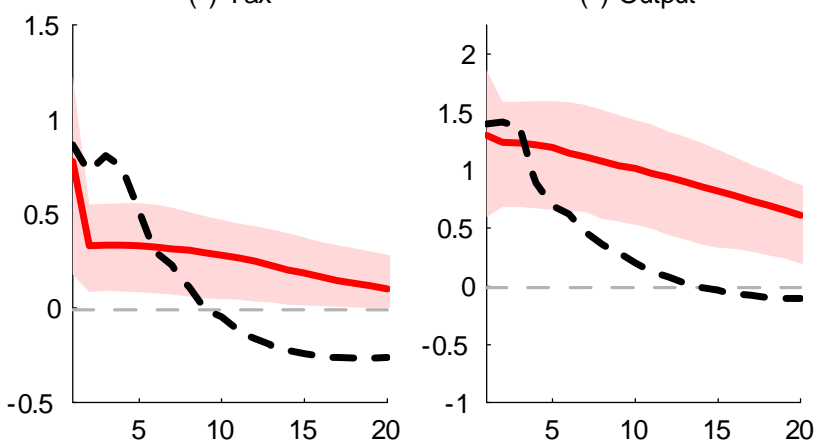

Figure 3: Recursive identification scheme: Impulse response functions (in percent) of government spending, government revenue ("Tax") and output to a government spending shock identified from a timing restriction. Estimation from a standard VAR (dashed-line) or from a GMA (plain line). The thin lines cover $90 \%$ of the posterior probability. For ease of comparison between the top and bottom panels, the responses to a contractionary shock are multiplied by -1 in the bottom panels. Estimation using quarterly data covering 1966-2014. 

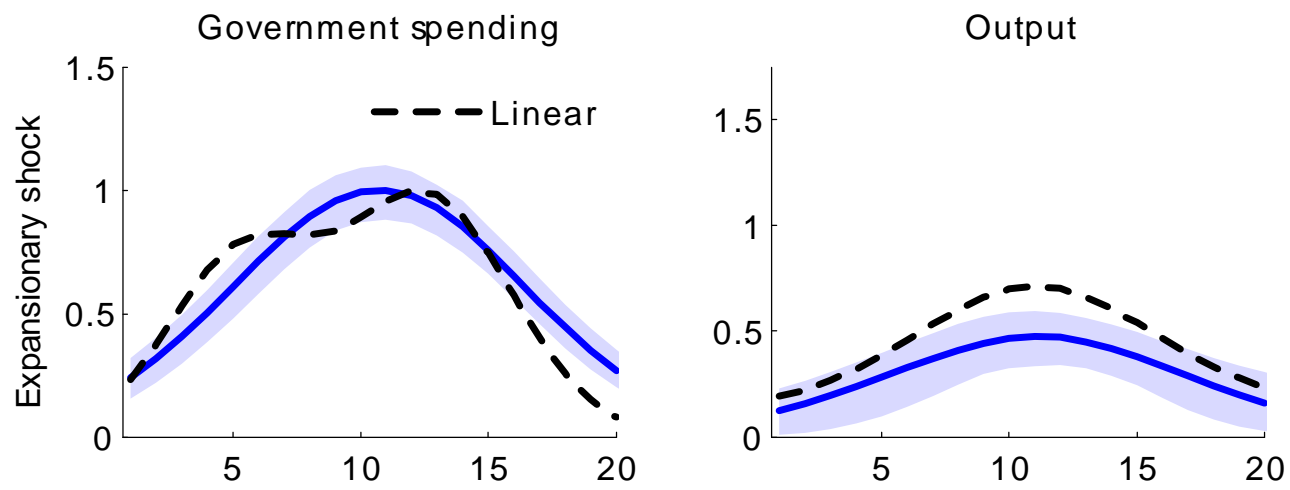

(-) Government spending

(-) Output
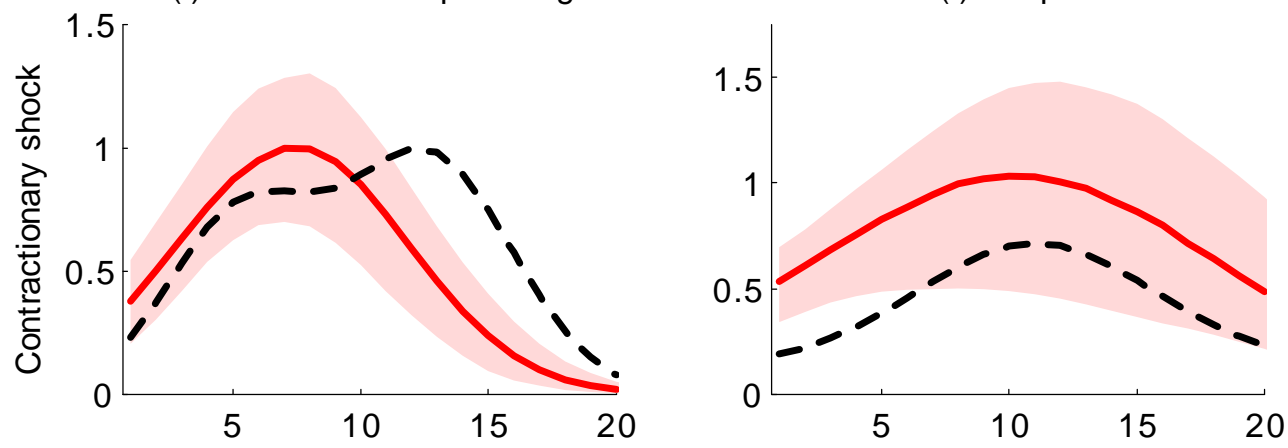

Figure 4: Narrative identification scheme: Impulse response functions (in percent) of government spending and output to a Ramey news shock identified from a narrative approach. Estimation from a linear GMA(2) (dashed-line) or from an asymmetric GMA(2) (plain line). The thin lines cover $90 \%$ of the posterior probability. For ease of comparison between the top and bottom panels, the responses to a contractionary shock are multiplied by -1 in the bottom panels. Estimation using quarterly data covering 1939-2014. 

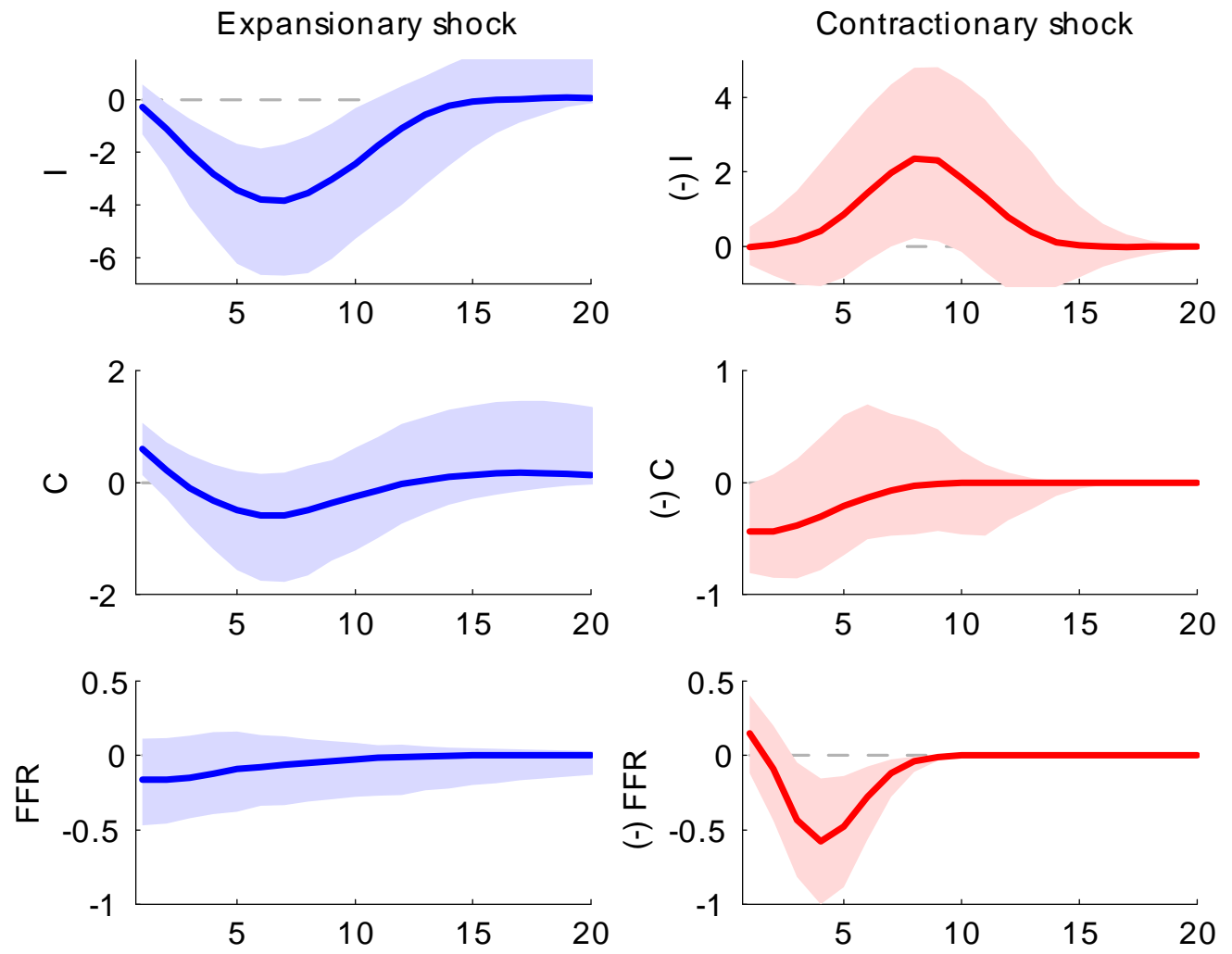

Figure 5: Recursive identification scheme: Impulse response functions (in percent) of Investment (I), Consumption (C) and the fed funds rate (FFR) following an expansionary government spending shock (left panel) and a contractionary shock (right panel). Estimates from a GMA(2) model using government spending shocks identified from a recursive ordering. For ease of comparison between the left and right panels, the responses to a contractionary shock are multiplied by -1 in the right panels. The shaded area covers $90 \%$ of the posterior probability. Estimation using quarterly data covering 1966-2014. 

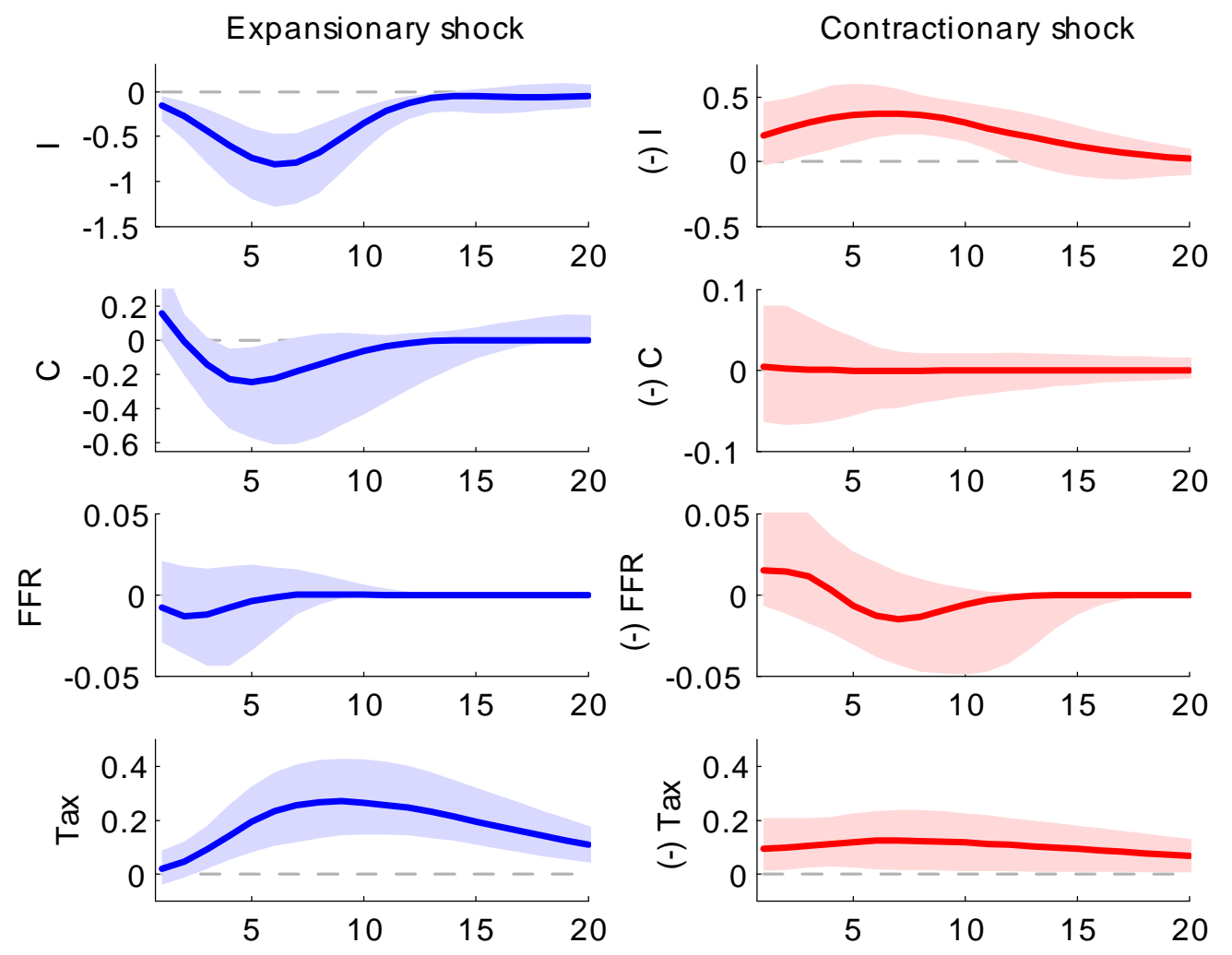

Figure 6: Narrative identification scheme: Impulse response functions (in percent) of Investment (I), Consumption (C), the fed funds rate (FFR) and the average marginal tax rate (Tax) following an expansionary Ramey news shock (left panel) and a contractionary Ramey news shock (right panel). Estimates from a GMA(2) model. For ease of comparison between the left and right panels, the responses to a contractionary shock are multiplied by -1 in the right panels. The shaded area covers $90 \%$ of the posterior probability. Estimation using quarterly data covering 1947-2014. 
Expansionary Shock
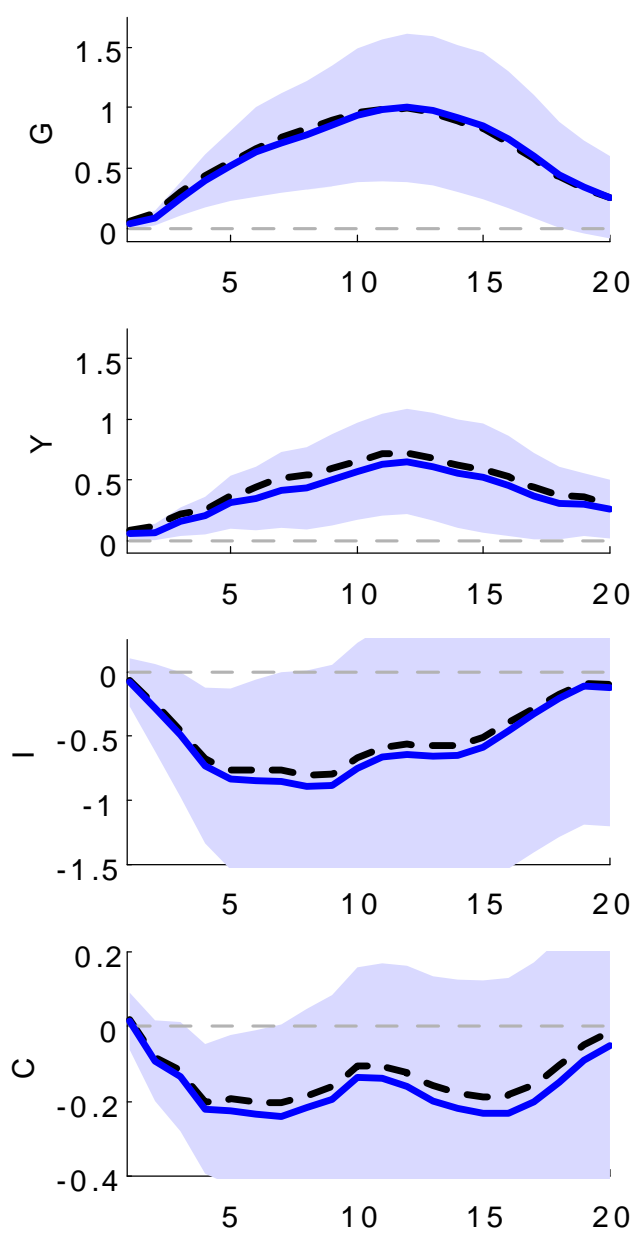

Contractionary Shock
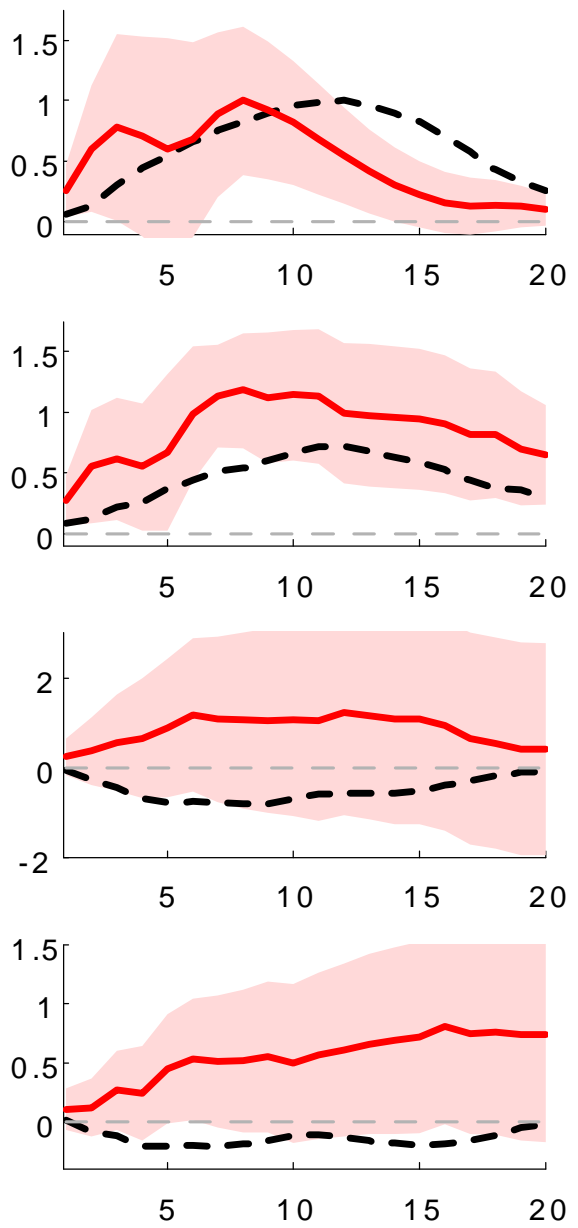

Figure 7: Narrative identification scheme and Local Projections: Impulse response functions (in percent) of Government Spending (G), Output (Y), Investment (I), and Consumption (C) following an expansionary Ramey news shock (left panel) and a contractionary Ramey news shock (right panel). Estimates from Local Projections. For ease of comparison between the left and right panels, the responses to a contractionary shock are multiplied by -1 in the right panels. The shaded areas are the 90 percent confidence bands calculated using Newey-West standard errors. Estimation using data covering 1939 to 2014 for G and Y and covering 1947 to 2014 for $\mathrm{C}$ and I. 


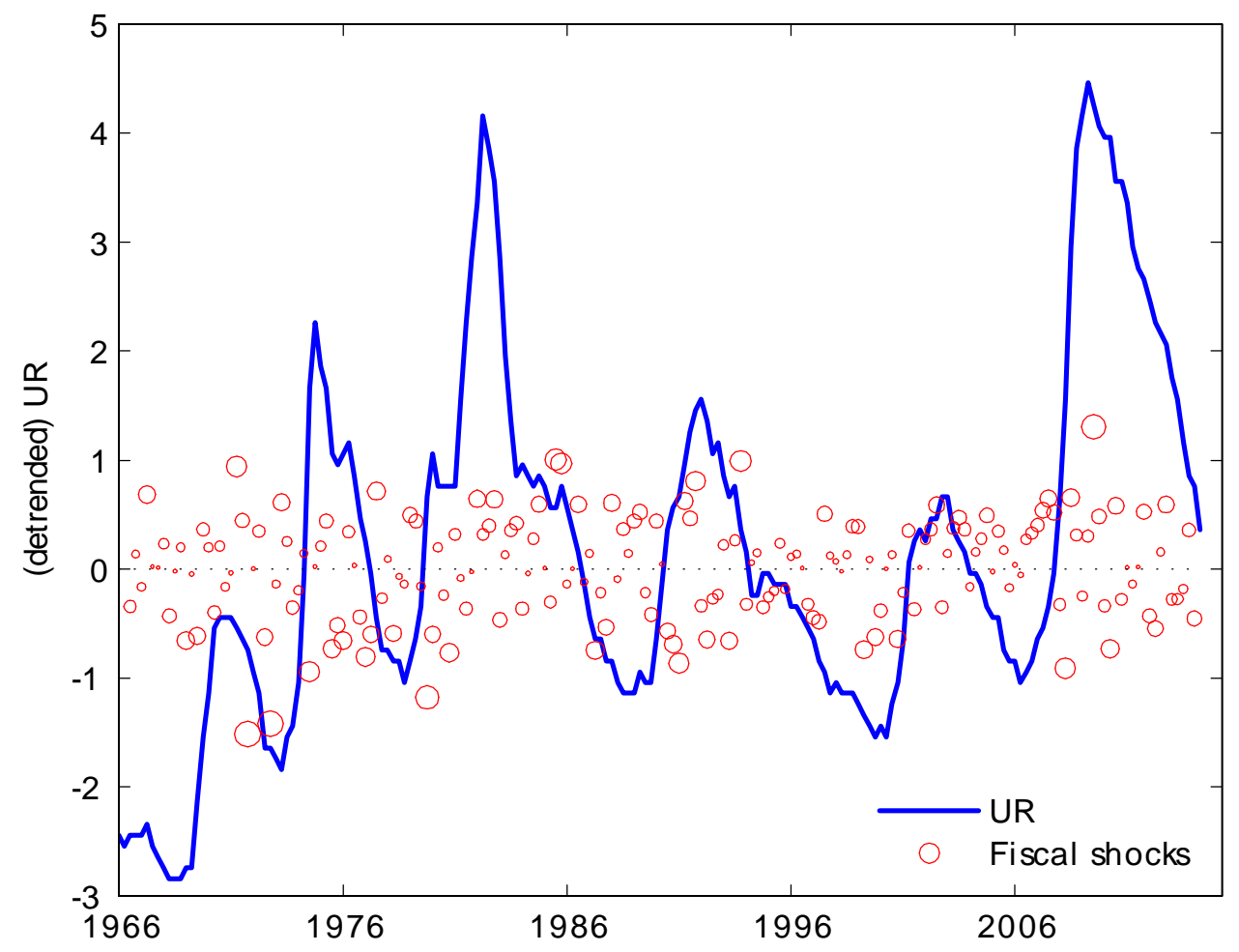

Figure 8: The business cycle indicator -the unemployment rate detrended with CBO's natural rate estimate- (solid line, left scale)-, and government spending shocks identified from a recursive ordering (circles, right scale) with larger circles indicating larger shocks. 

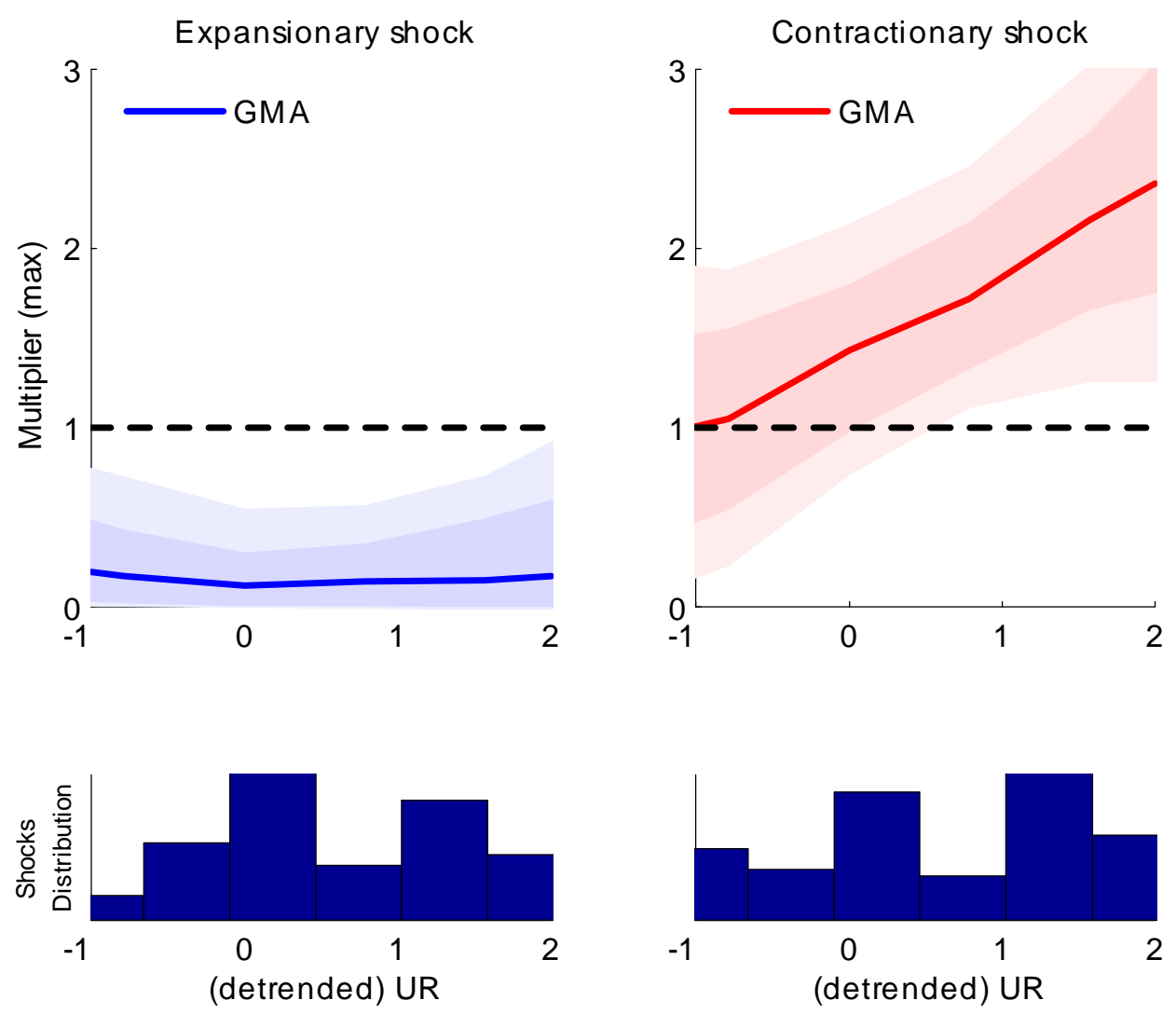

Figure 9: Recursive identification scheme: Size of the "max" multiplier as a function of the state of the business cycle (measured with detrended unemployment) for expansionary government spending shocks (left panel) and contractionary government spending shocks (right panel). The shaded areas respectively cover 68 and 90 percent of the posterior probability. The bottom panels plot the distributions of (respectively) contractionary shocks and expansionary shocks over the business cycle. Estimation using data covering 1966-2014. 

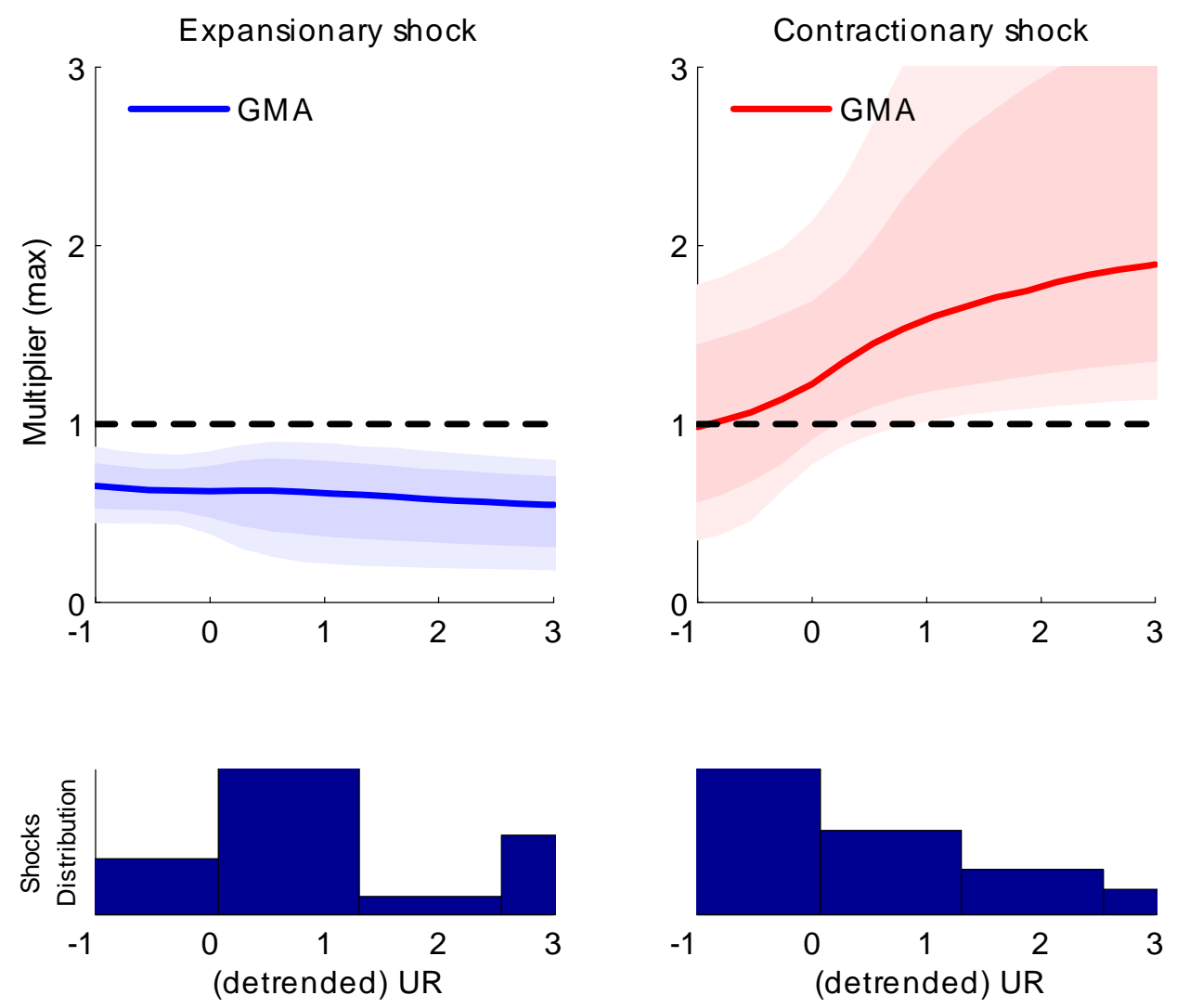

Figure 10: Narrative identification scheme: Size of the "max" multiplier as a function of the state of the business cycle (measured with detrended unemployment) for expansionary Ramey news shocks (left panel) and contractionary Ramey news shocks (right panel). The shaded areas respectively cover 68 and 90 percent of the posterior probability. The bottom panel plots the distribution of (respectively) contractionary shocks and expansionary shocks over the business cycle. Estimation using data covering 1966-2014. 

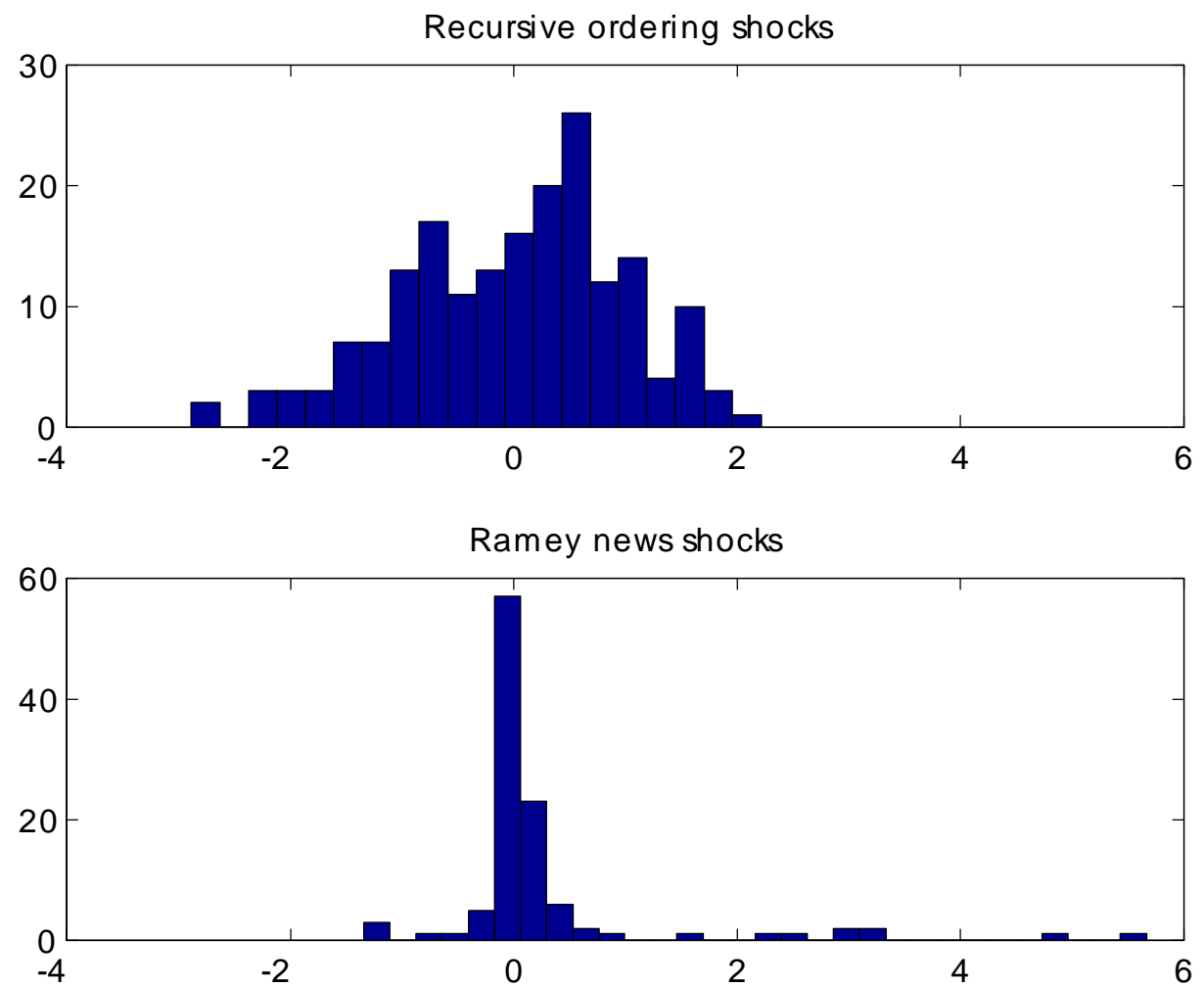

Figure 11: Histograms of the distributions of government spending shocks (rescaled by their standard-deviation). The upper-panel depicts the distribution of shocks recovered from a recursive ordering (1966-2014), the bottom-panel depicts the distribution of Ramey news shocks (1939-2014). 
Table 1: GMA estimates of government spending multipliers

\begin{tabular}{lccc|ccc}
\hline & \multicolumn{3}{c|}{ "Max" multiplier } & \multicolumn{3}{c}{ “Sum” multiplier } \\
\hline & Linear & $\begin{array}{c}\text { Expansionary } \\
\text { shock }\end{array}$ & $\begin{array}{c}\text { Contractionary } \\
\text { shock }\end{array}$ & Linear & $\begin{array}{c}\text { Expansionary } \\
\text { shock }\end{array}$ & $\begin{array}{c}\text { Contractionary } \\
\text { shock }\end{array}$ \\
\hline & & & & & & \\
AG shocks & 1.41 & 0.12 & 1.42 & 0.58 & -0.15 & 1.25 \\
$1966-2014$ & $(0.6--2.2)$ & $(-0.1--0.6)$ & $(0.8--2.2)$ & $(0.0-1.1)$ & $(-0.9--0.2)$ & $(0.7--1.8)$ \\
& & & & & & \\
$\begin{array}{l}\text { Ramey News } \\
\text { shocks }\end{array}$ & 0.72 & 0.49 & 1.03 & 0.73 & 0.51 & 1.76 \\
$1939-2014$ & $(0.6--0.8)$ & $(0.4--0.6)$ & $(0.6--1.6)$ & $(0.6--0.8)$ & $(0.3-0.6)$ & $(1.1--2.7)$ \\
\hline
\end{tabular}

Note: Estimates from GMA(2) models; i.e., using Gaussian Mixture Approximations of the impulse response functions with 2 Gaussians. Numbers in parenthesis cover $90 \%$ of the posterior probability. AG shocks refer to shocks obtained as in Auerbach and Gorodnichenlo (2012) from a Blanchard-Perotti recursive identification scheme augmented with professional forecasts of government spending. Ramey news shocks are the unexpected changes in anticipated future expenditures constructed by Ramey (2011). The "sum" multiplier is calculated by taking the integral of the impulse responses over the first 20 quarters. 